\title{
Pacific
}

Journal of

Mathematics

\section{A CONVEXITY THEOREM FOR SEMISIMPLE SYMMETRIC} SPACES

\author{
KARL-HERMANN NEEB
}




\title{
A CONVEXITY THEOREM FOR SEMISIMPLE SYMMETRIC SPACES
}

\section{Karl-HermanN NeEB}

\begin{abstract}
In this paper we prove a convexity theorem for semisimple symmetric spaces which generalizes Kostant's convexity theorem for Riemannian symmetric spaces. Let $\tau$ be an involution on the semisimple connected Lie group $G$ and $H=G_{0}^{\tau}$ the 1-component of the group of fixed points. We choose a Cartan involution $\theta$ of $G$ which commutes with $\tau$ and write $K=G^{\theta}$ for the group of fixed points. Then there exists an abelian subgroup $A$ of $G$, a subgroup $M$ of $K$ commuting with $A$, and a nilpotent subgroup $N$ such that $H M A N$ is an open subset of $G$ and there exists an analytic mapping $L: H M A N \rightarrow \mathfrak{a}=\mathbf{L}(A)$ with $L(h$ man $)=\log a$. The set of all elements in $A$ for which $a H \subseteq H M A N$ is a closed convex cone. Our main result is the description of the projections $L(a H) \subseteq \mathfrak{a}$ for these elements as the sum of the convex hull of the Weyl group orbit of $\log a$ and a certain convex cone in $\mathfrak{a}$.
\end{abstract}

0. Introduction. If $G$ is a connected semisimple Lie group and $G=K A^{\prime} N$ an Iwasawa decomposition, then the convexity theorem of Kostant describes the image of the sets $a K$ under the projection $G=K A^{\prime} N \rightarrow \mathfrak{a}^{\prime}=\mathbf{L}\left(A^{\prime}\right), k \exp X n \mapsto X$ as the convex hull of the Weyl group orbit through $\log a$. Recently van den Ban proved a generalization of this theorem to the following situation. Let $\tau$ be an involution on the semisimple Lie group $G$ with finite center, $G=$ $K A^{\prime} N$ a compatible Iwasawa decomposition, i.e., $K$ is $\tau$-invariant, and $\mathfrak{a}^{\prime}=\mathfrak{a}_{\mathfrak{h}}+\mathfrak{a}_{\mathfrak{q}}$ the corresponding decomposition of $\mathfrak{a}^{\prime}=\mathbf{L}\left(A^{\prime}\right)$ into 1 and -1 eigenspaces for $\tau$. Suppose that $H \subseteq G^{\tau}$ is an essentially connected subgroup (see $\S I$ for the definition). Then he describes the image of the sets $a H, a \in \exp \mathfrak{a}_{\mathfrak{q}}$ under the projection $F: G \rightarrow \mathfrak{a}_{\mathfrak{q}}$ defined by $g \in K \exp \left(\mathfrak{a}_{\mathfrak{h}}\right) \exp F(g) N$. This set is the sum of the convex hull of the orbit of $\log a$ under a certain Weyl group and a convex cone in $\mathfrak{a}_{\mathfrak{q}}$.

We generalize Kostant's theorem into another direction. We consider the projection $L: H M A N \rightarrow \mathfrak{a}$ defined by $g \in H M \exp L(g) N$, where $H \subseteq G^{\tau}$ is essentially connected and $M, A$, and $N$ are defined in $\S$ I. This makes sense because the $A$-component in a product hman is unique and $H M A N$ is open in $G$. So the main new difficulties are the non-compactness of $H$ and the fact that the projection $L$ 
is only defined on an open subset of $G$. Having identified the set of those elements in $A$ for which $L(a H)$ is defined, i.e., $a H \subseteq H M A N$, we describe the set $L(a H)$ as the sum of the Weyl group orbit through $\log a$ and a convex cone $C(a)$ in $a$. This description is very similar to the description in van den Ban's theorem. Nevertheless the result is of a different nature and so are the methods we use in the proof.

In the first section we start with the properties of the decomposition $H M A N$ and state the main theorem. Then we describe the set of elements $a \in A$ for which $a H \subseteq H M A N$ and reduce the problem to the case of regular symmetric spaces.

In $\S$ II we collect some facts about finite groups which are generated by reflections. For the applications in $§ I V$ we have to study the Weyl group orbits of non-compact convex sets and how their intersections with the chambers look like.

Section III provides some material about highest weight representations of Hermitian simple Lie groups, the holomorphic discrete series, and analytic continuation of the unitary representations to contraction representations of certain complex Lie semigroups. It turns out that we even need general bounded representations of certain Lie semigroups. These facts are used in $\S I V$ to prove one inclusion of the convexity theorem. The unitary highest weight representations replace the finite dimensional $K$-spherical representations which can be used to prove Kostant's theorem, and which were also used by van den Ban.

The fourth section contains the definitions concerning regular symmetric spaces and the proof of the convexity theorem. In addition to the material of $\S \S I I$ and III we have to use rank-1-reduction techniques to prove the other inclusion of the theorem. Fortunately we can use a great deal of the results for Riemannian symmetric spaces so we only have to consider the $\mathrm{Sl}(2, \mathbb{R})$-case in detail, which corresponds to the hyperboloid, an adjoint orbit of a hyperbolic element in the Lie algebras $(2, \mathbb{R})$.

The author thanks the referee for many helpful comments and for suggesting Proposition VI.7 and its proof.

I. Decompositions of a semisimple symmetric Lie group. In the following $G$ always denotes a connected real semisimple Lie group.

Definition I.1. A pair $(G, \tau)$ of a connected Lie group $G$ and an involutive automorphism $\tau$ is called a symmetric Lie group. We also write $\tau$ for the automorphism $d \tau(\mathbf{1})$ of the Lie algebra $\mathfrak{g}=\mathbf{L}(G)$ which is induced by $\tau$. Then $(\mathfrak{g}, \tau)$ is said to be a symmetric Lie algebra. We set 


$$
\begin{gathered}
G^{\tau}:=\{g \in G: \tau(g)=g\}, \\
\mathfrak{h}:=\{X \in \mathfrak{g}: \tau(X)=X\}, \quad \text { and } \mathfrak{q}:=\{X \in \mathfrak{g}: \tau(X)=-X\} .
\end{gathered}
$$

Since $G$ was assumed to be semisimple and connected, there exists a Cartan involution $\theta$ of $G$ and $\mathfrak{g}$ respectively such that $\tau \theta=\theta \tau$ ([Lo69, p. 153]). We set

$$
\mathfrak{k}:=\{X \in \mathfrak{g}: \theta(X)=X\}, \quad \mathfrak{p}:=\{X \in \mathfrak{g}: \theta(X)=-X\},
$$

and $K:=G^{\theta}=\langle\exp \mathfrak{k}\rangle$. Note that we have the direct vector space decomposition

$$
\mathfrak{g}=\mathfrak{h}_{\mathfrak{k}}+\mathfrak{h}_{\mathfrak{p}}+\mathfrak{q}_{\mathfrak{k}}+\mathfrak{q}_{\mathfrak{p}},
$$

where $\mathfrak{h}_{\mathfrak{k}}:=\mathfrak{h} \cap \mathfrak{k}, \mathfrak{h}_{\mathfrak{p}}:=\mathfrak{h} \cap \mathfrak{p}$, etc. We define the associated symmetric Lie algebra $\left(\mathfrak{h}^{a}, \tau^{a}\right)$ by $\mathfrak{h}^{a}:=\mathfrak{h}_{\mathfrak{k}}+\mathfrak{q}_{\mathfrak{p}}$ and $\tau^{a}:=\left.\tau\right|_{\mathfrak{h}^{a}}=\left.\theta\right|_{\mathfrak{h}^{a}}$. This is a reductive orthogonal symmetric Lie algebra ([Wa72, p. 42]). Now we choose $\mathfrak{a}$ maximal abelian in $\mathfrak{q}_{\mathfrak{p}}$ and $\mathfrak{a}^{\prime}$ maximal abelian in $\mathfrak{p}$ with $\mathfrak{a} \subseteq \mathfrak{a}^{\prime}$. Then the fact that the operators ad $X, X \in \mathfrak{a}$ are semisimple with real spectrum ([Hel78, p. 184]) implies that we have the root space decomposition

$$
\mathfrak{g}=Z_{\mathfrak{g}}(\mathfrak{a}) \oplus \bigoplus_{\alpha \in \Delta} \mathfrak{g}^{\alpha}
$$

where $\Delta=\Delta(\mathfrak{g}, \mathfrak{a})$ is the set of non-zero linear functionals on $\mathfrak{a}$ for which the root space $\mathfrak{g}^{\alpha}:=\{Y \in \mathfrak{g}:(\forall X \in \mathfrak{a})[X, Y]=\alpha(X) Y\}$ is non-zero. Set $\mathfrak{c}:=Z\left(\mathfrak{h}^{a}\right) \cap \mathfrak{q}_{\mathfrak{p}}$ and note that $\mathfrak{c} \subseteq \mathfrak{a}$. The set of compact roots is defined as

$$
\Delta_{k}:=\{\alpha \in \Delta: \alpha(\mathfrak{c})=\{0\}\},
$$

and $\Delta_{p}:=\Delta \backslash \Delta_{k}$ is the set of non-compact roots. We define a positive system $\Delta^{+}:=\left\{\alpha \in \Delta: \alpha\left(x_{0}\right)>0\right\}$ where $X_{0} \in \mathfrak{a}$ is an element with $\alpha\left(X_{0}\right) \neq 0$ for all $\alpha \in \Delta$, and $\alpha\left(X_{0}\right)<\beta\left(X_{0}\right)$ for all $\beta \in \Delta_{p}^{+}:=\Delta^{+} \cap \Delta_{p}$ and $\alpha \in \Delta_{k}^{+}:=\Delta^{+} \cap \Delta_{k}$. (One has to choose $X_{0}$ near to an element $X_{1}$ of $\mathfrak{c}$ where no non-compact root vanishes.) Next we write $\mathscr{W}$ for the Weyl group generated by the reflections $s_{\alpha}$ in the hyperplanes $\operatorname{ker} \alpha$ with $\mathfrak{g}^{\alpha} \cap \mathfrak{h}^{a} \neq\{0\}$. This is the Weyl group of the pair $\left(\mathfrak{h}^{a}, \mathfrak{a}\right)$. We define the minimal and maximal cones

$$
C_{\max }:=\left\{X \in \mathfrak{a}:\left(\forall \alpha \in \Delta_{p}^{+}\right) \alpha(X) \geq 0\right\}
$$

and

$$
C_{\text {min }}:=C_{\max }^{*}:=\left\{X \in \mathfrak{a}:\left(\forall Y \in C_{\max }\right) B(X, Y) \geq 0\right\},
$$


where $B(X, Y)=\operatorname{tr}(\operatorname{ad} X \operatorname{ad} Y)$ is the Cartan Killing form of $\mathfrak{g}$. Finally we define $\mathfrak{n}:=\bigoplus_{\alpha \in \Delta^{+}} \mathfrak{g}^{\alpha}, N:=\exp \mathfrak{n}, A:=\exp \mathfrak{a}$,

$$
M:=Z_{K}\left(\mathfrak{a}^{\prime}\right)=\left\{k \in K:\left.\operatorname{Ad}(k)\right|_{\mathfrak{a}^{\prime}}=\mathrm{id}_{\mathfrak{a}^{\prime}}\right\}
$$

and

$$
\mathfrak{m}:=\mathbf{L}(M)=Z_{\mathfrak{k}}\left(\mathfrak{a}^{\prime}\right)=\left\{X \in \mathfrak{k}:\left[X, \mathfrak{a}^{\prime}\right]=\{0\}\right\}
$$

The following proposition is a slight generalization of Proposition 1.10 in [OS80] to our general setting.

Proposition I.2. The following assertions hold:

(i) $\mathfrak{g}=\mathfrak{h}+\mathfrak{h}+\mathfrak{a}+\mathfrak{n}$.

(ii) $G_{0}^{\tau} M_{0} A N$ is an open subset of $G$.

(iii) $G^{\tau} \cap M A N=G^{\tau} \cap M$.

(iv) $\mathfrak{h} \cap(\mathfrak{m}+\mathfrak{a}+\mathfrak{n})=\mathfrak{h} \cap \mathfrak{m}$.

Proof. (i) From $\mathfrak{a} \subseteq \mathfrak{q}_{\mathfrak{p}}$ we conclude that $\tau\left(\mathfrak{g}^{\alpha}\right)=\mathfrak{g}^{-\alpha}$. For $X \in \mathfrak{g}^{-\alpha}$ and $\alpha \in \Delta^{+}$we therefore have that

$$
X=(X+\tau(X))-\tau(X) \in \mathfrak{h}+\mathfrak{n} .
$$

Since $\mathfrak{g}^{0}=\mathfrak{g}^{0} \cap \mathfrak{h}+\mathfrak{a}+\mathfrak{g}^{0} \cap \mathfrak{q}_{\mathfrak{k}}$ the assertion follows if we show that $\mathfrak{g}^{0} \cap \mathfrak{q}_{\mathfrak{k}} \subseteq \mathfrak{m}$. To see this, we first note that $\left[\mathfrak{a}, \mathfrak{a}^{\prime}\right]=0$ implies that $\left[\mathfrak{a}^{\prime}, \mathfrak{g}^{0}\right] \subseteq \mathfrak{g}^{0}$. Hence

$$
\left[\mathfrak{a}^{\prime}, \mathfrak{g}^{0} \cap \mathfrak{q}_{\mathfrak{k}}\right]=\left[\mathfrak{a}^{\prime} \cap \mathfrak{h}_{\mathfrak{p}}, \mathfrak{g}^{0} \cap \mathfrak{q}_{\mathfrak{k}}\right] \subseteq \mathfrak{g}^{0} \cap \mathfrak{q}_{\mathfrak{p}}=\mathfrak{a} .
$$

Therefore

$$
B\left(\mathfrak{a},\left[\mathfrak{a}^{\prime}, \mathfrak{g}^{0} \cap \mathfrak{q}_{\mathfrak{k}}\right]\right)=B\left(\left[\mathfrak{a}, \mathfrak{a}^{\prime}\right], \mathfrak{g}^{0} \cap \mathfrak{q}_{\mathfrak{k}}\right)=\{0\}
$$

and the positive definiteness of $B$ on $\mathfrak{a}$ imply that $\mathfrak{g}^{0} \cap \mathfrak{q}_{\mathfrak{k}} \subseteq Z_{\mathfrak{k}}\left(\mathfrak{a}^{\prime}\right)=$ $\mathfrak{m}$.

(ii) Set $P:=M A N$. It follows from (i) that $\mathfrak{k}+\mathbf{L}(P)=\mathfrak{g}$. We define the action of the group $G^{\tau} \times P$ on $G$ by $(h, p) . g:=h g p^{-1}$. Then the tangent space of the orbit of the subgroup $G_{0}^{\tau} \times P_{0}$ through $\mathbf{1}$ is $\mathfrak{h}+\mathbf{L}(P)=\mathfrak{g}$. Hence this orbit is open. Consequently the set $G^{\tau} P$ which is a union of translates of this open set is open, too.

(iii) Let $p=$ man $\in G^{\tau} \cap P$ with $m \in M, a \in A$, and $n \in N$. Then

$$
\text { man }=p=\tau(p)=\tau(m) a^{-1} \tau(n)
$$

and therefore $\tau(m)^{-1} m a^{2} n=\tau(n) \in M A N \cap \tau(N)$. We choose a positive system $\Delta\left(\mathfrak{g}, \mathfrak{a}^{\prime}\right)^{+}$of roots with respect to the maximal abelian subspace $\mathfrak{a}^{\prime} \subseteq \mathfrak{p}$ such that

$$
\Delta^{+}:=\left\{\left.\alpha\right|_{\mathfrak{a}}: \alpha \in \Delta\left(\mathfrak{g}, \mathfrak{a}^{\prime}\right)^{+}\right\}
$$


Then $\mathfrak{n} \subseteq \mathfrak{n}^{\prime}:=\bigoplus_{\alpha \in \Delta\left(\mathfrak{g}, \mathfrak{a}^{\prime}\right)^{+}} \mathfrak{g}^{\alpha}$ and the Bruhat decomposition of $G$ implies that

$$
M A N \cap \tau(N) \subseteq M A N^{\prime} \cap \theta\left(N^{\prime}\right)=\{\mathbf{1}\}
$$

([Wal88, p. 44]), whence $N=a=1$ and $\tau(m)=m$.

(iv) This follows from (iii) by considering the Lie algebra of the intersection of the two groups $G^{\tau}$ and $P$.

REMARK I.3. Note that the proof of Proposition I.2 shows that $G^{\tau} M A N$ is an open subset of $G$ which is a finite union of sets of the type $h G_{0}^{\tau} M_{0} A N m$, where $m$ runs through a set of representatives of $M / M_{0}$ and $h$ through a set of representatives of $G^{\tau} / G_{0}^{\tau}$ which is also a finite group ([Lo 69, p. 171]).

COROLLARY 1.4. The mapping

$$
L: G^{\tau} M A N \rightarrow \mathfrak{a}, \quad g=h \operatorname{man} \mapsto \log a
$$

is well defined and analytic.

Proof. If $g=h \operatorname{man}=h^{\prime} m^{\prime} a^{\prime} n^{\prime}$ with $h, h^{\prime} \in G^{\tau}, m, m^{\prime} \in M$, $a, a^{\prime} \in A$, and $n, n^{\prime} \in N$, then

$$
\begin{aligned}
h^{\prime-1} h & =\left(m^{\prime} a^{\prime} n^{\prime}\right)(m a n)^{-1} \\
& =m^{\prime} m^{-1} a^{\prime} a^{-1}\left((a m) n^{\prime} n^{-1}(a m)^{-1}\right) \in G^{\tau} \cap M A N \subseteq M
\end{aligned}
$$

(Proposition I.2) and therefore $a^{\prime}=a$ and $n^{\prime}=n$ follow from the uniqueness of the components in the decomposition $P:=M A N$. Again we consider the action of $G^{\tau} \times P$ on $G$ by $(h, p) . g=h g p^{-1}$. Then $\Omega^{\prime}:=G^{\tau} M A N$ is the open orbit through 1 and so the analytic structure induced on $\Omega^{\prime}$ from $G$ agrees with the analytic structure induced by the identification

$$
\Omega^{\prime} \cong\left(G^{\tau} \times P\right) /\left(G^{\tau} \times P\right)^{\mathbf{1}},
$$

where $\left(G^{\tau} \times P\right)^{1}=\left\{(h, h): h \in G^{\tau} \cap M\right\}$ is the stabilizer of 1 . The analyticity of the mapping $L$ now follows from the analyticity of the mapping

$$
\left(h,(\operatorname{man})^{-1}\right)=\left(h, n^{-1} a^{-1} m^{-1}\right) \mapsto \log a
$$

on $G^{\tau} \times P$ which is a consequence of the Iwasawa decomposition of $G$.

Definition I.5. An open subgroup $H \subseteq G^{\tau}$ is said to be essentially connected if $H=H_{0} Z_{K \cap H}(\mathfrak{a})$. 
We are interested in the sets $L(a H)$, where $H$ is an essentially connected open subgroup of $G^{\tau}$. Clearly these sets are well defined if and only if the set $a H$ is contained in $G^{\tau} M A N$. So we first have to study the set of those elements $a \in A$ for which this is true.

Definition I.6. An element $a \in A$ is called admissible if $a H \subseteq$ $G^{\tau} M A N$. The set of admissible elements is denoted $A_{\mathrm{adm}}$.

Now we have all definitions available which are necessary to state our main result.

TheOREM I.7 (The Convexity Theorem). Let $(G, \tau)$ be a connected semisimply symmetric Lie group, $H \subseteq G^{\tau}$ essentially connected, and $a \in A$ an admissible element. Then

$$
L(a H)=\operatorname{conv}(\mathscr{W} \log a)+C(a),
$$

where

$$
C(a)=\left\{Y \in C_{\min }:\left(\forall \alpha \in \Delta_{p}^{+}, \alpha(\mathscr{W} \log a)=\{0\}\right) \alpha(Y) \leq 0\right\} .
$$

Moreover, if $\Delta_{n}^{+}:=\left\{\alpha \in \Delta^{+}: \mathfrak{g}^{\alpha} \cap\left(\mathfrak{h}_{\mathfrak{p}}+\mathfrak{q}_{\mathfrak{k}}\right) \neq\{0\}\right\}$ and

$$
C_{n}:=\left\{Y \in \mathfrak{a}:\left(\forall \alpha \in \Delta_{n}^{+}\right) \alpha(X) \geq 0\right\},
$$

then

$$
\log A_{\mathrm{adm}}=\bigcap_{w \in \mathscr{W}} w\left(C_{n}\right)
$$

The proof of this theorem will be completed in $§ I V$. In the remainder of this section we reduce the result to an essential nonreducible case which will be proven in $§ I V$. We will also obtain more explicit descriptions of the cones $C(a)$ and $\mathfrak{a}_{\mathrm{adm}}:=\log A_{\mathrm{adm}}$.

REMARK I.8. It is clear that $1 \in A$ is always admissible. So $C(a)=$ $\{0\}$ and $C(a) \neq C_{\min }$ whenever $a=1$ and $C_{\min } \neq\{0\}$. Thus $C(a)$ cannot be replaced by the larger cone $C_{\min }$.

If $\mathfrak{g}$ is the smallest $\tau$-invariant ideal in $\mathfrak{g}$, i.e., if $(\mathfrak{g}, \tau)$ is irreducible, then we will see in Theorem I.20 that there are three cases. The Riemannian case, where $A_{\mathrm{adm}}=A$ and $C_{\min }=C(a)=\{0\}$ for all $a \in A$, the regular case, where $A_{\mathrm{adm}}=\exp \left(C_{\mathrm{max}}\right)$ and $C(a)=C_{\min }$ if and only if $a \neq 1$, and a third case where $A_{\mathrm{adm}}=\{1\}$ and $C(\mathbf{1})=C_{\min }=\{0\}$.

LEMMA I.9. Let $a \in A, H \subseteq G^{\tau}$ an essentially connected subgroup, and $\Omega:=G_{0}^{\tau} M_{0} A N$. Then the following are equivalent: 

(1) $a$ is admissible.
(2) $a H_{0} \subseteq \Omega$.
(3) $a \Omega \subseteq \Omega$.
(4) $a H_{0} a^{-1} \subseteq \Omega$.
(5) $a H a^{-1} \subseteq Z_{K \cap H}(\mathfrak{a}) \Omega$.
(6) $a H \subseteq H M A N$.

Proof. (1) $\Rightarrow(2)$ : If $a$ is admissible, then $a H_{0}$ is contained in the connected component of $G^{\tau} M A N$ which contains $a$. Hence $a H_{0} \subseteq$ $H_{0} M_{0} A N=\Omega$ according to Remark I.3.

(2) $\Rightarrow$ (3): If (2) is satisfied, then $a \Omega \subseteq \Omega M_{0} A N=\Omega$.

$(3) \Rightarrow(4)$ : This follows from

$$
a H_{0} a^{-1} \subseteq a \Omega a^{-1}=a \Omega \subseteq \Omega .
$$

(4) $\Rightarrow(5)$ : From $H=Z_{K \cap H}(\mathfrak{a}) H_{0}$ we conclude that

$$
a H a^{-1}=Z_{K \cap H}(\mathfrak{a}) a H_{0} a^{-1} \subseteq Z_{K \cap H}(\mathfrak{a}) \Omega .
$$

$(5) \Rightarrow(6)$ : This is a consequence of $H \Omega a=H \Omega \subseteq H M A N$.

$(6) \Rightarrow(1)$ : If $(6)$ holds, then

$$
a H \subseteq H M A N \subseteq G^{\tau} M A N
$$

shows that $a$ is admissible.

LEMMA I.10. If $a \in A$ is admissible, then $L(a H)=L\left(a H_{0}\right)$.

Proof. Since $L\left(h^{\prime} g a^{\prime}\right)=L(g)$ for every $g \in G^{\tau} M A N, h \in G^{\tau}$, and $a \in A$, we use $H=Z_{K \cap H}(\mathfrak{a}) H_{0}$ to see that

$$
L(a H)=L\left(a Z_{K \cap H}(\mathfrak{a}) H_{0}\right)=L\left(Z_{K \cap H}(\mathfrak{a}) a H_{0}\right)=L\left(a H_{0}\right) .
$$

REMARK I.11. In view of Lemmas I.9 and I.10 the essential case is when $H$ is connected. One may say that the essential connectedness of $H$ insures that the disconnectedness of $H$ causes no additional difficulties.

Next we reduce the convexity theorem to the case where $Z(G)=\{1\}$ and $\operatorname{Ad}(G) \cong G$.

LEMMA I.12. If the Convexity Theorem holds for the adjoint group $\operatorname{Ad}(G)$ with respect to the involution

$$
\tilde{\tau}: \operatorname{Ad}(g) \mapsto \tau \operatorname{Ad}(g) \tau=\operatorname{Ad}(\tau(g)),
$$

then it holds for $G$. 
Proof. According to Remark I.11 we may assume that $H$ is connected. Then

$$
\operatorname{Ad}(\Omega)=\operatorname{Ad}(H)_{0} \operatorname{Ad}(M)_{0} \operatorname{Ad}(A) \operatorname{Ad}(N)=\widetilde{\Omega},
$$

where $\widetilde{\Omega}$ is the corresponding subset in the symmetric group $\operatorname{Ad}(G)$. Let $a \in A$ be admissible. Then $a \Omega \subseteq \Omega$ (Lemma I.9) and therefore

$$
\operatorname{Ad}(a) \widetilde{\Omega}=\operatorname{Ad}(a \Omega) \subseteq \operatorname{Ad}(\Omega)=\widetilde{\Omega}
$$

It follows that $\operatorname{Ad}(a)$ is admissible in $\operatorname{Ad}(G)$ (Lemma I.9). For the following we note that $\Omega$ is the connected component of the unit element in the open set $G^{\tau} M A N$. If $\operatorname{Ad}(a)$ is admissible, then

$$
a H \subseteq a \Omega \subseteq \operatorname{Ad}^{-1} \widetilde{\Omega}=\Omega Z(G)=\Omega,
$$

and therefore $a$ is admissible. Consequently $\mathrm{Ad}_{A}$ maps the set of admissible elements in $A$ bijectively onto the set of admissible elements in $\operatorname{Ad}(A)$.

From $a h \in H M \exp L(a h) N$ we deduce that

$$
\operatorname{Ad}(a) \operatorname{Ad}(h) \in \operatorname{Ad}(H) \operatorname{Ad}(M) e^{\operatorname{ad} L(a h)} \operatorname{Ad}(N),
$$

so that the function

$$
\widetilde{L}: \operatorname{Ad}(\Omega) \rightarrow \mathfrak{a} \text { defined by } g \in \operatorname{Ad}(H M) \exp (\widetilde{L}(g)) \operatorname{Ad}(N)
$$

satisfies the relation

$$
\widetilde{L}(\operatorname{Ad}(g))=\operatorname{ad}(L(g))
$$

This proves that

$$
\widetilde{L}(\operatorname{Ad}(a) \operatorname{Ad}(H))=\operatorname{ad}(L(a H))
$$

Since the cones $C(a)$ only depend on the Lie algebra, the Convexity Theorem for $\operatorname{Ad}(G)$ implies the Convexity Theorem for $G$ because ad is an isomorphism of Lie algebras.

According to the preceding lemma we may assume that $Z(G)=$ $\{1\}$. This assumption implies in particular that $G$ is a direct product of its simple factors. 
LEMMA I.13. The simple ideals of $\mathfrak{g}$ are invariant under $\theta$.

Proof. Let $\mathfrak{g}=\bigoplus_{i=1}^{n} \mathfrak{g}_{i}$ be a decomposition of $\mathfrak{g}$ into simple ideals and $\theta_{i}$ a Cartan involution of $\mathfrak{g}_{i}$. Then $\tilde{\theta}:=\bigoplus_{i=1}^{n} \theta_{i}$ is a Cartan involution of $\mathfrak{g}$ which preserves the simple ideals. According to [Hel78, p. 183] there exists $g \in G$ with $\theta=\tilde{\theta} \circ \operatorname{Ad}(g)$. Thus $\theta$ preserves the simple ideals because this holds for $\operatorname{Ad}(g)$ and $\tilde{\theta}$.

Lemma I.14. Suppose that $Z(G)=\{1\}$. If the Convexity Theorem holds for all minimal connected $\tau$-invariant normal subgroups of $G$, then it holds for $G$. Moreover, $G \cong \prod_{i=1}^{n} G_{i}$, where the $G_{i}$ are the minimal connected $\tau$-invariant normal subgroups, $A=$ $\prod_{i=1}^{n}\left(A \cap G_{i}\right), A_{\mathrm{adm}}=\prod_{i=1}^{n}\left(A_{\mathrm{adm}} \cap G_{i}\right)$, and if $a=a_{1} \cdots a_{n} \in$ $A_{\mathrm{adm}}$, then

$$
C(a)=\sum_{i=1}^{n} C\left(a_{i}\right)
$$

Proof. Let $\mathfrak{g}=\bigoplus_{i=1}^{n} \mathfrak{g}_{i}$ be a decomposition into minimal $\tau$-invariant ideals. Then $\mathfrak{h}=\bigoplus_{i=1}^{n} \mathfrak{h}_{i}$, where $\mathfrak{h}_{i}:=\mathfrak{h} \cap \mathfrak{g}_{i}$, and similarly $\mathfrak{q}=$ $\bigoplus_{i=1}^{n} \mathfrak{q}_{i}, \mathfrak{k}=\bigoplus_{i=1}^{n} \mathfrak{k}_{i}$, and $\mathfrak{p}=\bigoplus_{i=1}^{n} \mathfrak{p}_{i}$ (Lemma I.13). If we choose $\mathfrak{a}_{i}$ maximal abelian in $\mathfrak{q}_{\mathfrak{p}} \cap \mathfrak{g}_{i}$, then $\tilde{\mathfrak{a}}:=\bigoplus_{i=1}^{n} \mathfrak{a}_{i}$ is maximal abelian in $\mathfrak{q}_{\mathfrak{p}}$, and $\mathfrak{a}$ is conjugate to $\tilde{\mathfrak{a}}$ under $e^{\text {ad } \mathfrak{h}_{\mathfrak{e}}}([\mathrm{Hel78}, \mathrm{p} .247]$ or [Ne91b, 2.9]). Hence $\mathfrak{a}=\bigoplus_{i=1}^{n}\left(\mathfrak{a} \cap \mathfrak{g}_{i}\right)$. The same argument and the fact that $\mathfrak{p}=\bigoplus_{i=1}^{n} \mathfrak{p}_{i}$ imply that every maximal abelian subspace $\mathfrak{a}^{\prime} \subseteq \mathfrak{p}$ with $\mathfrak{a} \subseteq \mathfrak{a}^{\prime}$ satisfies $\mathfrak{a}^{\prime}=\bigoplus_{i=1}^{n}\left(\mathfrak{a}^{\prime} \cap \mathfrak{g}_{i}\right)$. All these facts together prove that $G \cong \prod_{i=1}^{n} G_{i}, H=\prod_{i=1}^{n}\left(H \cap G_{i}\right), K=\prod_{i=1}^{n}\left(K \cap G_{i}\right), M=$ $\prod_{i=1}^{n}\left(M \cap G_{i}\right), A=\prod_{i=1}^{n}\left(A \cap G_{i}\right)$, and that $N=\prod_{i=1}^{n}\left(N \cap G_{i}\right)$, where $G_{i}:=\left\langle\exp \mathfrak{g}_{i}\right\rangle$. This entails that

$$
H M A N=\prod_{i=1}^{n}\left(H \cap G_{i}\right)\left(M \cap G_{i}\right)\left(A \cap G_{i}\right)\left(N \cap G_{i}\right),
$$

and $a=a_{1} \cdots a_{n}$ with $a_{i} \in A \cap G_{i}$ is admissible if and only if $a_{i}$ is admissible in $A \cap G_{i}$ (Lemma I.9).

In addition, we have that

$$
C_{\min }=\sum_{i=1}^{n}\left(C_{\min } \cap \mathfrak{g}_{i}\right), \quad \log a=\sum_{i=1}^{n} \log a_{i},
$$

and

$$
\mathscr{W} \log a=\sum_{i=1}^{n} \mathscr{W} \log a_{i}
$$


where $\mathscr{W} \log a_{i}$ agrees with the Weyl group orbit under the Weyl group $\mathscr{W}_{i}$ associated to the ideal $\mathfrak{g}_{i}$. This is the group generated by the reflections $s_{\alpha}$ with $\mathfrak{g}^{\alpha} \cap\left(\mathfrak{h}^{a} \cap \mathfrak{g}_{i}\right) \neq\{0\}$.

Suppose that $a$ is admissible. Then the definition of $C(a)$ and the preceding paragraph show that

$$
C(a)=\sum_{i=1}^{n} C\left(a_{i}\right) .
$$

Thus, under the assumption that the Convexity theorem holds for the factors $G_{i}$, we find that

$$
\begin{aligned}
L(a H) & =\sum_{i=1}^{n} L\left(a_{i}\left(H \cap G_{i}\right)\right) \\
& =\sum_{i=1}^{n}\left(\operatorname{conv} \mathscr{W}\left(\log a_{i}\right)+c\left(a_{i}\right)\right) \\
& =\sum_{i=1}^{n} \operatorname{conv} \mathscr{W}\left(\log a_{i}\right)+\sum_{i=1}^{n} C\left(a_{i}\right) \\
& =\operatorname{conv}(\mathscr{W} \log a)+C(a) .
\end{aligned}
$$

This lemma implies in particular that we can restrict our attention to irreducible symmetric Lie algebras, i.e., $\mathfrak{g}$ is a minimal non-zero $\tau$-invariant ideal of $\mathfrak{g}$. We note that this implies in particular that $\mathfrak{h}=[\mathfrak{q}, \mathfrak{q}]$ because $\mathfrak{q}+[\mathfrak{q}, \mathfrak{q}]$ is an ideal of $\mathfrak{g}$ (the case where $\mathfrak{h}=\mathfrak{g}$ is trivial). In the remainder of this section we also assume that $Z(G)=$ $\{1\}$ which is justified by Lemma I.12. We set $H^{a}:=\left\langle\exp \mathfrak{h}^{a}\right\rangle=G_{0}^{\theta \tau}$. This is a closed connected subgroup of $G$.

The crucial idea to classify the essentially different situations in the irreducible case is to consider the semigroup $S_{\Omega}$.

Definition I.15. We set $S_{\Omega}:=\{g \in G: g \Omega \subseteq \Omega\}$, where $\Omega=$ $G_{0}^{\tau} M_{0} A N$.

Corollary I.16. $S_{\Omega} \cap A$ is the set of admissible elements in $A$.

Proof. This follows from Lemma I.7 and the definition on $S_{\Omega}$.

The following proposition is a crucial ingredient in the classification of the irreducible situations. 
Proposition I.17. Let $G$ be a connected reductive Lie group with the Cartan decomposition $G=K \exp \mathfrak{p}$, where $Z(G) \subseteq K$, and $S \subseteq G$ a subsemigroup which contains $K$. Then $S$ is a connected closed subgroup which is a product $S=K G_{1}$, where $G_{1}$ is a connected normal subgroup.

Proof. Since $Z(G) \subseteq K$ we have that $S=Z(G)\left(S \cap G^{\prime}\right)$ and $G^{\prime}=$ $\left(K \cap G^{\prime}\right) \exp \mathfrak{p}$ is a Cartan decomposition of $G^{\prime}$. Hence we may assume that $G$ is semisimple. Let $\mathfrak{a} \subseteq \mathfrak{p}$ be a maximal abelian subspace, $C:=$ $\exp ^{-1}(S) \cap \mathfrak{a}$, and $\mathscr{W}:=N_{K}(\mathfrak{a}) / Z_{K}(\mathfrak{a})$ the Weyl group. It follows from $G=K A K$ ([Hel78, p. 402]), where $A:=\exp \mathfrak{a}$, that $S=K(A \cap S) K$ and therefore that $C \neq\{0\}$ if and only if $S \neq K$. We assume this. Then $C=\exp ^{-1}(S \cap A) \cap \mathfrak{a}$ is a $\mathscr{W}$-invariant subsemigroup of $\mathfrak{a}$. We will prove that $C$ is a vector subspace of $\mathfrak{a}$.

Sublemma. If $\alpha \in \Delta(\mathfrak{g}, \mathfrak{a})$ and $X \in C$ with $\alpha(X) \neq 0$, then $C$ contains the line segment

$$
\left\{2 X, X+s_{\alpha}(X)\right\}=\left\{Y \in \mathfrak{a}: Y-2 X \in[0,1]\left(s_{\alpha}(X)-X\right)\right\} .
$$

Proof. Choose $X_{\alpha} \in \mathfrak{g}^{\alpha} \backslash\{0\}$ and set

$$
\begin{gathered}
Y_{\alpha}:=X_{\alpha}+\theta\left(X_{\alpha}\right), \quad Z_{\alpha}:=\left[X_{\alpha}, \theta X_{\alpha}\right], \quad \text { and } \\
\mathfrak{g}_{\alpha}:=\operatorname{span}\left\{X_{\alpha}, \theta X_{\alpha}, Z_{\alpha}\right\} .
\end{gathered}
$$

Then $Z_{\alpha} \in \mathfrak{a}$ because $Z_{\alpha} \in\left[\mathfrak{g}^{\alpha}, \mathfrak{g}^{-\alpha}\right] \cap \mathfrak{p}=Z_{\mathfrak{p}}(\mathfrak{a})=\mathfrak{a}$. Therefore $\mathfrak{g}_{\alpha} \cong \operatorname{sl}(2, \mathbb{R})$ is a three dimensional simple subalgebra with

$$
\begin{gathered}
\mathfrak{g}_{\alpha} \cap \mathfrak{k}=\mathbb{R} Y_{\alpha}, \quad \mathfrak{g}_{\alpha} \cap \mathfrak{a}=\mathbb{R} Z_{\alpha}, \quad \text { and } \\
\mathfrak{g}_{\alpha} \cap \mathfrak{p}=\operatorname{span}\left\{Z_{\alpha}, X_{\alpha}-\theta X_{\alpha}\right\}
\end{gathered}
$$

([Hel78, p. 407]). Moreover $\alpha\left(Z_{\alpha}\right) \neq 0$ and by interchanging $\alpha$ and $-\alpha$ we may assume that $\alpha\left(Z_{\alpha}\right) \alpha(X)>0$. By rescaling of $X_{\alpha}$ we may even assume that $X_{0}:=X-Z_{\alpha}$ satisfies $\alpha\left(X_{0}\right)=0$. This entails that $\left[X_{0}, \mathfrak{g}_{\alpha}\right]=\{0\}$. We define $\beta(t) \in \mathbb{R}^{+}$by

$$
\exp \left(Z_{\alpha}\right) \exp \left(e^{\operatorname{ad} t Y_{\alpha}} Z_{\alpha}\right) \in K_{\alpha} \exp \left(\beta(t) Z_{\alpha}\right) K_{\alpha},
$$

where $K_{\alpha}=\exp \mathbb{R} Y_{\alpha} \subseteq K$. Then $\beta(0)=2$ and there exists $t \in$ $\mathbb{R}^{+}$with $\beta(t)=0$. It follows that $[0,2] \subseteq \beta([0, t])$ because $\beta$ is continuous and $[0,1]$ is connected. Now

$$
\exp (X) \exp \left(e^{\operatorname{ad} t Y_{\alpha}} X\right) \in S S \subseteq S
$$


and therefore

$$
\begin{aligned}
\exp (X) \exp \left(e^{\left.\operatorname{ad} t Y_{\alpha} X\right)}\right. & =\exp \left(Z_{\alpha}\right) \exp \left(X_{0}\right) \exp \left(e^{\operatorname{ad} t Y_{\alpha}} Z_{\alpha}\right) \exp \left(X_{0}\right) \\
& =\exp \left(Z_{\alpha}\right) \exp \left(e^{\operatorname{ad} t Y_{\alpha}} Z_{\alpha}\right) \exp \left(2 X_{0}\right) \\
& \in K_{\alpha} \exp \left(\beta(t) Z_{\alpha}\right) K_{\alpha} \exp \left(2 X_{0}\right) \\
& =K_{\alpha} \exp \left(\beta(t) Z_{\alpha}+2 X_{0}\right) K_{\alpha}
\end{aligned}
$$

Hence $\beta(t) Z_{\alpha}+2 X_{0} \in C$ for all $t \in \mathbb{R}$ and in particular

$$
C \supseteq 2 X_{0}+[0,2] Z_{\alpha}=\left\{2 X, 2 X_{0}\right\}=\left\{2 X, X+s_{\alpha}(X)\right\} .
$$

We continue with the proof of Proposition I.17. Let $X \in C$ be arbitrary and set $E:=\operatorname{span} \mathscr{W} X$. For every $\beta \in \Delta(\mathfrak{g}, \mathfrak{a})$ with $\beta(E) \neq$ $\{0\}$ there exists $w_{0} \in \mathscr{W}$ such that $\beta\left(w_{0} . X\right) \neq 0$. Then the sublemma shows that

$$
\left\{2 w_{0} . X, w_{0} . X+s_{\beta}\left(w_{0} . X\right)\right\} \subseteq C .
$$

Set $Z:=\sum_{w \in \mathscr{W}} w \cdot X$. Then $w . Z=Z$ for all $w \in \mathscr{W}$ and therefore $\alpha(Z)=0$ for all $\alpha \in \Delta(\mathfrak{g}, \mathfrak{a})$ because $s_{\alpha}(Z)=Z$ for all such $\alpha$. Hence $Z=0$ because $\mathfrak{g}$ is semisimple. Consequently

$$
\begin{aligned}
{[0,1] } & \left(s_{\beta}\left(w_{0} \cdot X\right)-w_{0} \cdot X\right) \\
& \subseteq\left\{2 w_{0} \cdot X, w_{0} \cdot X+s_{\beta}\left(w_{0} \cdot X\right)\right\}+\sum_{w \in \mathscr{W} \backslash\left\{w_{0}\right\}} 2 w \cdot X \\
& \subseteq \sum_{w \in \mathscr{W}} C \subseteq C
\end{aligned}
$$

for every $\beta$ with $\beta(E) \neq\{0\}$. But $\sum_{w \in \mathscr{W}} w \cdot X=0$ implies that

$$
\begin{aligned}
E & =\operatorname{span}\{2 . Y-Y: Y \in E, w \in \mathscr{W}\} \\
& =\operatorname{span}\left\{w^{\prime} .(w . X)-w . X: w, w^{\prime} \in \mathscr{W}\right\} \\
& =\operatorname{span}\left\{s_{\beta}(w . X)-w . X: \beta \in \Delta(\mathfrak{g}, \mathfrak{a}), w \in \mathscr{W}, \beta(w . X) \neq 0\right\} .
\end{aligned}
$$

Thus $C$ contains a generating simplex of $E$, and in particular $\operatorname{int}_{E}(C)$ $\neq \varnothing$. Let $Y \in C \cap E$ be an inner point. Then

$$
0=\sum_{w \in \mathscr{W}} w \cdot Y \in \operatorname{int}_{E}(C)
$$

shows that $E \subseteq C$. Since $X \in C$ was arbitrary, we have proved that $C$ is a $\mathscr{W}$-invariant subspace of a. Hence $S=K \exp (C) K$ is a closed connected subgroup of $G$ which is invariant under conjugation with $K$. Let $\mathfrak{g}=\bigoplus_{i=1}^{n} \mathfrak{g}_{i}$ be a decomposition into simple ideals and $\mathfrak{p}_{i}:=\mathfrak{p} \cap \mathfrak{g}_{i}$. Then $\mathfrak{p}=\bigoplus_{i=1}^{n} \mathfrak{p}_{i}$ is the decomposition of $\mathfrak{p}$ into simple $\mathfrak{k}$-modules ([Hel78, p. 379]). Therefore $\mathbf{L}(S)=\mathfrak{k}+\sum_{\mathfrak{p}_{\imath} \cap \mathbf{L}(S) \neq\{0\}} \mathfrak{g}_{i}$ and the assertion follows. 
CoRollary I.18. If $G$ is a connected simple Lie group, $G=K \exp \mathfrak{p}$ a Cartan decomposition, and $S$ a subsemigroup containing $K$, then either $S=K$ or $S=G$.

In the classification of the irreducible cases (Theorem I.20) we will use some sl(2)-reduction arguments. So we have to consider this case first.

EXAMPLE I.19. We consider the Lie algebra $\mathfrak{g}=\operatorname{sl}(2, \mathbb{R})$ and the group $G=\operatorname{Sl}(2, \mathbb{R})$ with the involution $\tau$ defined by conjugation with the matrix $\left(\begin{array}{ll}0 & 1 \\ 1 & 0\end{array}\right)$. Then

$$
\tau\left(\begin{array}{rr}
a & b \\
c & -a
\end{array}\right)=\left(\begin{array}{rr}
-a & c \\
b & a
\end{array}\right)
$$

and the elements

$$
X=\left(\begin{array}{ll}
0 & 1 \\
0 & 0
\end{array}\right), \quad Y=\left(\begin{array}{ll}
0 & 0 \\
1 & 0
\end{array}\right), \quad \text { and } Z=\left(\begin{array}{rr}
1 & 0 \\
0 & -1
\end{array}\right)
$$

satisfy

$Y=\tau X, \quad Z=[X, Y], \quad \mathfrak{h}=\mathbb{R}(X+Y), \quad \mathfrak{a}=\mathbb{R} Z, \quad$ and $\quad \mathfrak{g}^{\alpha}=\mathbb{R} X$, where $\alpha(Z)=2$.

The corresponding groups are

$$
H=\left\{\left(\begin{array}{cc}
\cosh t & \sinh t \\
\sinh t & \cosh t
\end{array}\right): t \in \mathbb{R}\right\}, \quad A=\left\{\left(\begin{array}{cc}
e^{t} & 0 \\
0 & e^{-t}
\end{array}\right): t \in \mathbb{R}\right\},
$$

$K=\mathrm{SO}(2)$, and

$$
N=\left\{\left(\begin{array}{ll}
1 & t \\
0 & 1
\end{array}\right): t \in \mathbb{R}\right\}, \quad M=\{\mathbf{1},-\mathbf{1}\}=Z_{K}(\mathfrak{a}) .
$$

The formula

$$
\begin{aligned}
& L(\exp (s(X+Y)) \exp (t Z) \exp (u X)) \\
& \quad=L\left(\begin{array}{ll}
e^{t} \cosh s & e^{t} u \cosh s+e^{-t} \sinh s \\
e^{t} \sinh s & e^{t} u \sinh s+e^{-t} \cosh s
\end{array}\right)=t Z
\end{aligned}
$$

implies that the analytic function $L: H A N \rightarrow \mathfrak{a}$ is given by

$$
L\left(\begin{array}{ll}
a & b \\
c & d
\end{array}\right)=\frac{1}{2} \log \left(a^{2}-c^{2}\right) Z
$$

and the open subset $H A N \subseteq G$ is described by the inequality $a^{2}-$ $c^{2}>0$. 
In particular we have that

$$
\begin{aligned}
& L\left(\exp \left(t_{0} Z\right) \exp (s(X+\tau X))\right)=L\left(\begin{array}{cc}
e^{t_{0}} \cosh s & e^{t_{0}} \sinh s \\
e^{-t_{0}} \sinh s & e^{-t_{0} \cosh s}
\end{array}\right) \\
& \quad=\frac{1}{2} \log \left(e^{2 t_{0}} \cosh ^{2}(s)-e^{-2 t_{0}} \sinh ^{2}(s)\right) Z \\
& =\left(t_{0}+\frac{1}{2} \log \left(\cosh ^{2}(s)-e^{-4 t_{0}} \sinh ^{2}(s)\right)\right) Z \\
& =\left(t_{0}+\frac{1}{2} \log \left(1+\left(1-e^{-4 t_{0}}\right) \sinh ^{2}(s)\right)\right) Z
\end{aligned}
$$

The same argument as in the proof of Lemma I.12 shows that this formula holds for all connected Lie groups which are locally isomorphic to $\operatorname{Sl}(2, \mathbb{R})$. We observe that for $t_{0}<0$ this analytic function of $s$ has no extension to the whole set $\exp \left(t_{0} Z\right) H$.

THEOREM I.20. If $(\mathfrak{g}, \tau)$ is an irreducible semisimple symmetric Lie algebra, then one of the following assertions hold:

(i) $(\mathfrak{g}, \tau)$ is orthogonal, i.e., $\tau=\theta, \mathfrak{h}^{a}=\mathfrak{g}, A_{\mathrm{adm}}=A$, and $C_{\min }=\{0\}$.

(ii) $Z\left(\mathfrak{h}^{a}\right) \subseteq \mathfrak{h}_{\mathfrak{k}}, A_{\mathrm{adm}}=\{\mathbf{1}\}$, and $C_{\min }=\{0\}$.

(iii) $Z\left(\mathfrak{h}^{a}\right) \cap \mathfrak{q}_{\mathfrak{p}} \neq\{0\}, \mathfrak{g}^{c}:=\mathfrak{h}+i \mathfrak{q}$ is a semisimple Hermitian Lie algebra and $\mathfrak{g}$ is simple.

Proof. First we note that the condition that $\mathfrak{c}=Z\left(\mathfrak{h}^{a}\right) \cap \mathfrak{q}_{\mathfrak{p}}=\{0\}$ entails that $\Delta_{p}=\varnothing$ and therefore that $C_{\min }=\{0\}$. This applies in the case (i) and (ii).

Moreover, (i) holds precisely when $\mathfrak{g}=\mathfrak{h}^{a}$. So we assume that $\mathfrak{g} \neq \mathfrak{h}^{a}$ and that $Z\left(\mathfrak{h}^{a}\right) \subseteq \mathfrak{h}_{\mathfrak{k}}$. We have to show that $A_{\text {adm }}=\{\mathbf{1}\}$. We consider the reductive subgroup $H^{a}=\left\langle\operatorname{exph} \mathfrak{h}^{a}\right\rangle$ of $G$. Then the restriction of $\tau$ to $H^{a}$ is a Cartan involution and

$$
H^{a}=(H \cap K)_{0} \exp \mathfrak{q}_{\mathfrak{p}}
$$

is a Cartan decomposition. Moreover the assumption $Z\left(\mathfrak{h}^{a}\right) \cap \mathfrak{q}_{\mathfrak{p}}=$ $\{0\}$ implies that $Z\left(H^{a}\right) \subseteq(H \cap K)_{0}$. Set $S:=S_{\Omega} \cap H^{a}$. Then $S$ is a subsemigroup of $H^{a}$ which contains $(H \cap K)_{0}$ and therefore Proposition I.17 applies. Hence $S$ is a closed subgroup of $H^{a}$.

Set $C:=\mathfrak{a} \cap \mathbf{L}(S)$. Suppose that there exists $\alpha \in \Delta(\mathfrak{g}, \mathfrak{a})$ with $\mathfrak{g}^{\alpha} \nsubseteq \mathfrak{h}^{a}$ and $Z \in C$ with $\alpha(Z) \neq\{0\}$. From $\mathfrak{a} \subseteq \mathfrak{h}^{a}=\{X \in$ $\mathfrak{g}: \theta \tau(X)=X\}$ we conclude that $\theta \tau\left(\mathfrak{g}^{\alpha}\right)=\mathfrak{g}^{\alpha}$ and there exists $X_{\alpha} \in$ $\mathfrak{g}^{\alpha} \backslash\{0\}$ such that $\theta \tau\left(X_{\alpha}\right)=-X_{\alpha}$, i.e., $\tau\left(X_{\alpha}\right)=-\theta\left(X_{\alpha}\right)$. Again we set $\mathfrak{g}_{\alpha}:=\operatorname{span}\left\{X_{\alpha}, \theta X_{\alpha},\left[X_{\alpha}, \theta X_{\alpha}\right]\right\}$. This is a three dimensional subalgebra isomorphic to $\operatorname{sl}(2, \mathbb{R})$ which is invariant under $\theta$ and $\tau$. 
We set $\mathfrak{k}_{\alpha}:=\mathbb{R}\left(X_{\alpha}+\theta X_{\alpha}\right), \mathfrak{h}_{\alpha}:=\mathbb{R}\left(X_{\alpha}-\theta X_{\alpha}\right)=\mathbb{R}\left(X_{\alpha}+\tau X_{\alpha}\right), \mathfrak{a}_{\alpha}:=$ $\mathbb{R}\left[X_{\alpha}, \theta X_{\alpha}\right], K_{\alpha}:=\left\langle\exp \mathfrak{k}_{\alpha}\right\rangle, H_{\alpha}:=\exp \mathfrak{h}_{\alpha}$, and $A_{\alpha}:=\exp \mathfrak{a}_{\alpha}$. Now $H_{\alpha} \subseteq H_{0} \subseteq S_{\Omega}$ and therefore $X_{\alpha}+\tau X_{\alpha} \in \mathbf{L}\left(S_{\Omega}\right)$. Moreover $Z \in C$ implies that $\mathbb{R} Z \subseteq \mathbf{L}(S) \subseteq \mathbf{L}\left(S_{\Omega}\right):=\left\{X \in \mathfrak{g}: \exp \mathbb{R}^{+} X \subseteq S_{\Omega}\right\}$ and therefore the subalgebra generated by $Z$ and $X_{\alpha}+\tau X_{\alpha}$ is contained in $\mathbf{L}\left(S_{\Omega}\right)$ ([HHL89, II.1.8, IV.1.27]). From

$$
\left[Z, X_{\alpha}+\tau X_{\alpha}\right]=\alpha(Z)\left(X_{\alpha}-\tau X_{\alpha}\right) \neq 0
$$

we conclude that this subalgebra contains $\mathfrak{g}_{\alpha}$. Hence $G_{\alpha}:=\left\langle\exp \mathfrak{g}_{\alpha}\right\rangle \subseteq$ $S_{\Omega} \subseteq H M A N$. It follows from the uniqueness of the function

$$
L_{\alpha}: H_{\alpha} A_{\alpha} N_{\alpha} \rightarrow \mathfrak{a}_{\alpha}, \quad h a n \mapsto \log a
$$

that $L_{\alpha}=\left.L\right|_{H_{\alpha} A_{\alpha} N_{\alpha}}$. We normalize $X_{\alpha}$ such that the element $Z_{\alpha}:=$ $\left[X_{\alpha}, \tau X_{\alpha}\right]$ satisfies the relation $\alpha\left(Z_{\alpha}\right)=2$. Then Example I.19 shows that

$$
\begin{aligned}
& L\left(\exp \left(t Z_{\alpha}\right) \exp \left(s\left(X_{\alpha}+\tau X_{\alpha}\right)\right)\right) \\
& \quad=\left(t+\frac{1}{2} \log \left(1+\left(1-e^{-4 t}\right) \sinh ^{2}(s)\right)\right) Z_{\alpha} .
\end{aligned}
$$

For $t<0$ this contradicts the fact that $L$ is an analytic mapping defined on the whole set $\exp \left(t Z_{\alpha}\right) H_{\alpha} \subseteq \Omega$, whence $\mathfrak{g}^{\alpha} \subseteq \mathfrak{h}^{a}$ whenever $\alpha(C) \neq\{0\}$.

Set $\mathfrak{g}_{1}:=\mathbf{L}\left(S_{\Omega} \cap S_{\Omega}^{-1}\right)$ and $\mathfrak{a}_{1}:=C=\mathfrak{g}_{1} \cap \mathfrak{a}$. Then this implies that

$$
\mathfrak{g}_{1}=\mathfrak{g}_{1}^{0} \oplus \bigoplus_{\alpha \in \Delta\left(\mathfrak{g}_{1}, \mathfrak{a}_{1}\right)} \mathfrak{g}_{1}^{\alpha} \subseteq \mathfrak{g}_{1}^{0}+\mathfrak{h}^{a} .
$$

We note that the two subspaces $\mathfrak{g}_{1}^{0}$ and $\mathfrak{h}^{a}$ are invariant under the involution $\theta \tau$. Therefore $\mathfrak{h}_{\mathfrak{p}} \subseteq \mathfrak{g}_{1}$ shows that

$$
\mathfrak{h}_{\mathfrak{p}} \subseteq\left\{X \in \mathfrak{g}_{1}: \theta \tau(X)=-X\right\} \subseteq \mathfrak{g}_{1}^{0} .
$$

Moreover the $\theta \tau$-invariance of $H, M, A$, and $N$ entails that $\theta \tau(\Omega)$ $=\Omega$, and therefore that $\theta \tau\left(S_{\Omega}\right)=S_{\Omega}$. So $\theta \tau\left(\mathfrak{g}_{1}\right)=\mathfrak{g}_{1}$ implies that $\theta\left(\mathfrak{g}_{1}\right)=\mathfrak{g}_{1}$. This leads to the decomposition

$$
\mathfrak{g}_{1}=\mathfrak{h}_{\mathfrak{k}}+\mathfrak{h}_{\mathfrak{p}}+\mathfrak{q}_{\mathfrak{k}, 1}+\mathfrak{q}_{\mathfrak{p}, 1},
$$

where $\mathfrak{q}_{\mathfrak{k}, 1}=\mathfrak{q}_{\mathfrak{k}} \cap \mathfrak{g}_{1}, \mathfrak{g}_{\mathfrak{p}, 1}=\mathfrak{q}_{\mathfrak{p}} \cap \mathfrak{g}_{1}$, and $\left(\mathfrak{h}_{1}^{a},\left.\theta\right|_{\mathfrak{h}_{1}} ^{\alpha}\right)$ with $\mathfrak{h}_{1}^{a}:=\mathfrak{h}_{\mathfrak{k}}+\mathfrak{q}_{\mathfrak{p}, 1}$ is an orthogonal symmetric Lie algebra. In particular we have that $\mathfrak{q}_{\mathfrak{p}, 1}=e^{\operatorname{ad}_{\mathfrak{k}}} \mathfrak{a}_{1} \quad\left(\left[\right.\right.$ Hel78, p. 247] or [Ne91b, 2.9]). So $\left[\mathfrak{h}_{\mathfrak{k}}, \mathfrak{h}_{\mathfrak{p}}\right] \subseteq \mathfrak{h}_{\mathfrak{p}}$ implies that

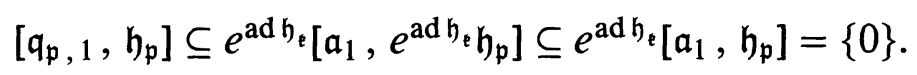


Together with the $\mathfrak{h}_{\mathfrak{k}}$-invariance of $\mathfrak{q}_{\mathfrak{p}, 1}$ this proves the $\mathfrak{h}$-invariance of $\mathfrak{q}_{\mathfrak{p}, 1}$. Set $\mathfrak{g}_{2}:=\mathfrak{q}_{\mathfrak{p}, 1}+\left[\mathfrak{q}_{\mathfrak{p}, 1}, \mathfrak{q}_{\mathfrak{p}, 1}\right]$. Then $\mathfrak{g}_{2}$ is an $\mathfrak{h}$-invariant subalgebra of $\mathfrak{g}$. We claim that $\mathfrak{g}_{2}$ is an ideal of $\mathfrak{g}$. First

$$
B\left(\mathfrak{h}_{\mathfrak{p}},\left[\mathfrak{q}_{\mathfrak{p}, 1}, \mathfrak{q}_{\mathfrak{k}}\right]\right)=B\left(\left[\mathfrak{h}_{\mathfrak{p}}, \mathfrak{q}_{\mathfrak{p}, 1}\right], \mathfrak{q}_{\mathfrak{k}}\right)=\{0\} \quad \text { and } \quad\left[\mathfrak{q}_{\mathfrak{p}}, 1, \mathfrak{q}_{\mathfrak{k}}\right] \subseteq \mathfrak{h}_{\mathfrak{p}}
$$

entail that $\left[\mathfrak{q}_{\mathfrak{k}}, \mathfrak{q}_{\mathfrak{p}, 1}\right]=\{0\}$ and hence that $\left[\mathfrak{q}_{\mathfrak{k}}, \mathfrak{g}_{2}\right]=\{0\}$. We write $\mathfrak{q}_{\mathfrak{p}, 1}^{\perp}$ for the orthogonal complement of $\mathfrak{q}_{\mathfrak{p}, 1}$ in $\mathfrak{q}_{\mathfrak{q}}$. Then $\left[\mathfrak{q}_{\mathfrak{p}, 1}^{\perp}, \mathfrak{q}_{\mathfrak{p}, 1}\right.$ ] $\subseteq \mathfrak{h}_{\mathfrak{k}}$ and

$$
B\left(\mathfrak{h}_{\mathfrak{k}},\left[\mathfrak{q}_{\mathfrak{p}, 1}, \mathfrak{q}_{\mathfrak{p}, 1}^{\perp}\right]\right)=B\left(\left[\mathfrak{h}_{\mathfrak{k}}, \mathfrak{q}_{\mathfrak{p}, q}\right], \mathfrak{q}_{\mathfrak{p}, 1}^{\perp}\right) \subseteq B\left(\mathfrak{q}, 1, \mathfrak{q}_{\mathfrak{p}, 1}^{\perp}\right)=\{0\} .
$$

Therefore $\left[\mathfrak{q}_{\mathfrak{p}, 1} \mathfrak{q}_{\mathfrak{p}, 1}^{\perp}\right]=\{0\}$ and consequently $\left[\mathfrak{q}_{\mathfrak{p}, 1}^{\perp}, \mathfrak{g}_{2}\right]=\{0\}$. So $\mathfrak{g}_{2}$ is a $\tau$-invariant ideal of $\mathfrak{g}$. Since $(\mathfrak{g}, \tau)$ is assumed to be irreducible, there are two possibilities. The first one is $\mathfrak{g}=\mathfrak{g}_{2}, \mathfrak{h}=\left[\mathfrak{q}_{\mathfrak{p}, 1}, \mathfrak{q}_{\mathfrak{p}, 1}\right]=\mathfrak{k}$ and $\mathfrak{q}=\mathfrak{q}_{\mathfrak{p}, 1}=\mathfrak{p}$. This is impossible if $\mathfrak{h}^{a} \neq \mathfrak{g}$. So $\mathfrak{g}_{2}=\{0\}$, $\mathfrak{q}_{\mathfrak{p}, 1}=\{0\}, \mathfrak{a}_{1}=\{0\}$, and finally $A_{\mathrm{adm}}=\{\mathbf{1}\}$.

The remaining case is $Z\left(\mathfrak{h}^{a}\right) \cap \mathfrak{q}_{\mathfrak{p}} \neq\{0\}$. It is clear that $Z\left(\mathfrak{h}^{a}\right)=$ $Z\left(\mathfrak{h}^{a}\right) \cap \mathfrak{h}_{\mathfrak{k}}+Z\left(\mathfrak{h}^{a}\right) \cap \mathfrak{q}_{\mathfrak{p}}$ and therefore

$$
i Z\left(\mathfrak{h}^{a}\right) \cap \mathfrak{q}_{\mathfrak{p}} \subseteq Z\left(\mathfrak{h}_{\mathfrak{k}}+i \mathfrak{q}_{\mathfrak{p}}\right) .
$$

Set $\mathfrak{k}^{c}:=\mathfrak{h}_{\mathfrak{k}}+i \mathfrak{q}_{\mathfrak{p}}$. This is a maximal compactly embedded subalgebra of the dual symmetric Lie algebra $\mathfrak{g}^{c}:=\mathfrak{h}+i \mathfrak{q}$. If $\mathfrak{g}$ is not simple, then $\mathfrak{g} \cong \mathfrak{g}_{1} \oplus \mathfrak{g}_{1}$, where' $\tau$ acts by $\tau(X, Y)=(Y, X)$ and $\mathfrak{g}^{c} \cong$ $\left(\mathfrak{g}_{1}\right)_{\mathbb{C}}=\mathfrak{g}_{1}+i \mathfrak{g}_{1}$, where $\mathfrak{g}_{1}$ is a simple real Lie algebra. If $\mathfrak{g}_{1}=\mathfrak{k}_{1}+\mathfrak{p}_{1}$ is a Cartan decomposition of $\mathfrak{g}_{1}$, then $\mathfrak{k}_{1}+i \mathfrak{p}_{1} \cong \mathfrak{h}_{\mathfrak{k}}+i \mathfrak{q}_{\mathfrak{p}}=\mathfrak{k}^{c}$ is a compact real form of the complex Lie algebra $\mathfrak{g}^{c}$. It follows in particular that $Z\left(\mathfrak{k}^{c}\right)=\{0\}$ which is a contradiction. Consequently $\mathfrak{g}$ is simple. Moreover $\left(\mathfrak{g}^{c}, \tau\right)$ is an irreducible symmetric Lie algebra and there are two cases:

(1) $\mathfrak{g}^{c}$ is simple. Then $Z\left(\mathfrak{k}^{c}\right) \neq\{0\}$ implies that $\mathfrak{g}^{c}$ is a Hermitian simple Lie algebra.

(2) $\mathfrak{g}^{c} \cong \mathfrak{h} \oplus \mathfrak{h}$ and $\mathfrak{g} \cong \mathfrak{h}_{\mathbb{C}}$, where $\mathfrak{q}=i \mathfrak{h}$. Then $\mathfrak{k}^{c}=\mathfrak{h}_{\mathfrak{k}}=i \mathfrak{h}_{\mathfrak{k}}$ and therefore $Z\left(\mathfrak{h}_{\mathfrak{k}}\right) \neq\{0\}$. It follows that $\mathfrak{h}$ is a simple Hermitian Lie algebra.

Remark I.21. In Case (i) the Convexity Theorem reduces to Kostant's Convexity Theorem ([Hel84, p. 476])

$$
\mathbf{L}(a K)=\operatorname{conv}(\mathscr{W} \log a) \quad \forall a \in A
$$

because $K=H, C(a)=C_{\min }=\{0\}$, and $A_{\mathrm{adm}}=A$.

In Case (ii) the statement

$$
\mathbf{L}(a H)=\operatorname{conv}(\mathscr{W} \log a)+C(a) \quad \forall a \in A_{\mathrm{adm}}
$$


of the Convexity Theorem reduces to

$$
\mathbf{L}(H)=\{0\}
$$

because $C(\mathbf{1})=C_{\min }=\{0\}$ and $A_{\mathrm{adm}}=\{\mathbf{1}\}$.

Summing up the reductions of this section it remains to prove the Convexity Theorem only in Case (iii) of Theorem I.20 and when $H$ is connected and $Z(G)=\{1\}$. This will be done in Section IV, where we will also prove the general formula for $A_{\mathrm{adm}}$ and show that $A_{\mathrm{adm}}=$ $\exp \left(C_{\max }\right)$ in this case. For $a \in A_{\text {adm }}$ we will see that

$$
C(a)= \begin{cases}C_{\min } & \text { if } a \neq \mathbf{1}, \\ \{0\} & \text { if } a=\mathbf{1}\end{cases}
$$

II. Groups generated by reflections. In this section $E$ denotes the finite dimensional Euclidean vector space, $\mathscr{H}$ is a finite set of codimension-one subspaces, and $\mathscr{W}$ the subgroup of the orthogonal group generated by the reflections in the hyperplanes from $\mathscr{H}$. We make the following assumptions:

(A1) The group $\mathscr{W}$ leaves the set $\mathscr{H}$ invariant.

(A2) $\mathscr{W}$ is finite.

Definition II.1. A connected component $C$ of $E \backslash \bigcup \mathscr{H}$ is called a chamber. If $C$ is a chamber, then we associate to $C$ a system $E_{\mathscr{H}, C}=\left\{e_{H}: H \in \mathscr{H}\right\}$ of unit vectors defined by

$$
e_{H}^{\perp}=H \quad \text { and } \quad\left\langle e_{H}, Y\right\rangle>0(\forall Y \in C) .
$$

For a subset $F \subseteq E$ we define the dual cone

$$
F^{*}:=\{Y \in E:(\forall X \in F)\langle X, Y\rangle \geq 0\} .
$$

Proposition II.2. Let $C \subseteq E$ be a chamber. Then the following assertions hold:

(i) $\bar{C}$ is a fundamental domain for the action of the group $\mathscr{W}$, i.e., every $\mathscr{W}$-orbit meets $\bar{C}$ in exactly one point.

(ii) If $X \in C$ and $Y \in C$, then

$$
\langle X, Y\rangle \geq\langle X, s(Y)\rangle \quad \forall s \in \mathscr{W} .
$$

Proof. (i) We note that our assumptions imply that (D1) and ( $\left.\mathrm{D}^{\prime} 2\right)$ in [Bou81, Ch. V, §3] are satisfied. Therefore (i) is Theorem 2 in [Bou81, Ch. V, §3, 3.3]. 
(ii) It follows from the proof of Lemma 2 in [Bou81, Ch. V, $\S 3,3.1]$ that

Therefore

$$
\|X-Y\| \leq\|X-s(Y)\| \quad \forall s \in \mathscr{W}
$$

$$
\begin{aligned}
\|X-Y\|^{2} & =\|X\|^{2}+\|Y\|^{2}-2\langle X, Y\rangle \\
& \leq\|X\|^{2}+\|s(Y)\|^{2}-2\langle X, s(Y)\rangle
\end{aligned}
$$

and the assertion follows from the orthogonality of $s \in \mathscr{W}$. $C^{*}$.

Corollary II.3. For $Y \in \bar{C}$ and $s \in \mathscr{W}$ we have that $Y-s(Y) \in$

Definition II.4. Let $F \subseteq E$ be a non-empty closed convex subset. We denote with

$$
\operatorname{co}(F):=\overline{\operatorname{conv}(\mathscr{W}(F))}
$$

the closed convex hull of the $\mathscr{W}$-orbit of $F$. For $F=\{X\}$ we also write $\operatorname{co}(X):=\operatorname{co}(\{X\})$.

From now on $C$ denotes a fixed chamber in $E$.

LEMMA II.5. Let $F \subseteq \bar{C}$ be a closed convex subset and $X \in F$. Then the following assertions hold:

(i) $\operatorname{co}(F) \subseteq \overline{F-C^{*}}$.

(ii) $\left(X-C^{*}\right) \cap \bar{C} \subseteq \operatorname{co}(X)$.

(iii) $\left(F-C^{*}\right) \cap \bar{C} \subseteq \operatorname{co}(F) \cap \bar{C} \subseteq \overline{F-C^{*}} \cap \bar{C}$.

(iv) $\bigcap_{s \in \mathscr{W}} s\left(F-C^{*}\right) \subseteq \operatorname{co}(F) \subseteq \bigcap_{s \in \mathscr{W}} s\left(\overline{F-C^{*}}\right)$.

Proof. (i) Since $\overline{F-C^{*}}$ is obviously closed and convex, it suffices to show that $\mathscr{W}(F) \subseteq \overline{F-C^{*}}$. Let $X \in F$ and $s \in \mathscr{W}$. If $s(X) \notin$ $\overline{F-C^{*}}$, then by the Hahn-Banach Theorem, there exists $Y \in E$ such that

$$
\langle s(X), Y\rangle>\sup \left\{\langle Y, Z\rangle: Z \in \overline{F-C^{*}}\right\} .
$$

In particular the functional $f: Z \mapsto\langle Y, Z\rangle$ is bounded from above on the set $X-C^{*}$. Consequently $Y \in-\left(-C^{*}\right)^{*}=\bar{C}$. Using Corollary II. 3 we find that

$$
\left.\langle X, Y\rangle \geq\left\langle X, s^{-1}(Y)\right\rangle=\langle s(X), Y\rangle\right\rangle\langle X, Y\rangle
$$

because $X \in F-C^{*}$. This contradiction shows that $\mathscr{W}(X) \subseteq \overline{F-C^{*}}$.

(ii) If (ii) is false, then there exist $Y \in E$ and $Z \in C^{*}$ such that $X-Z \in \bar{C}$ and

$$
\langle Y, X-Z\rangle>\langle Y, s(X)\rangle \quad \forall s \in \mathscr{W}
$$


Select $\sigma \in \mathscr{W}$ with $\sigma^{-1}(Y) \in \bar{C}$ (Proposition II.2). Then, since the relations

$$
Z \in C^{*} \text { and }(X-Z)-\sigma^{-1}(X-Z) \in C^{*}
$$

(Corollary II.3) imply by addition that

$$
X-\sigma^{-1}(X-Z) \in C^{*},
$$

we obtain

$$
\left\langle\sigma^{-1}(Y), X-\sigma^{-1}(X-Z)\right\rangle \geq 0,
$$

i.e., $\langle Y, \sigma(X)-X+Z\rangle \geq 0$. Hence

$$
\langle Y, \sigma(X)\rangle \geq\langle Y, X-Z\rangle,
$$

a contradiction.

(iii) The inclusion

$$
\operatorname{co}(F) \cap \bar{C} \subseteq \overline{F-C^{*}} \cap \bar{C}
$$

follows from (i). The other inclusion follows from (ii) because

$$
\left(F-C^{*}\right) \cap \bar{C}=\bigcup_{X \in F}\left(X-C^{*}\right) \cap \bar{C} \subseteq \bigcup_{X \in F} \operatorname{co}(X) \subseteq \operatorname{co}(F) .
$$

(iv) The $\mathscr{W}$-invariance of $\operatorname{co}(F)$ and (i) shows that

$$
\operatorname{co}(F) \subseteq \bigcap_{s \in \mathscr{W}} s\left(\overline{F-C^{*}}\right) .
$$

If, conversely, $X \in \bigcap_{s \in \mathscr{W}} s\left(F-C^{*}\right)$, then the set $\mathscr{W}(X)$ meets $\bar{C}$ in a point of $F-C^{*}$ (Proposition II.2). Now

$$
X \in \mathscr{W}\left(\left(F-C^{*}\right) \cap \bar{C}\right) \subseteq \mathscr{W}(\operatorname{co}(F))=\operatorname{co}(F)
$$

is a consequence of (iii).

REMARK II.6. Note that the inclusions in (iii) and (iv) in Lemma II.5 are equalities if the set $F-C^{*}$ is closed. This is true if $F$ is compact and in particular if $F=\{X\}$ consists of a single point. In the latter case Lemma II.5 specializes to Lemma 8.3 in [Hel84].

Next we show that $F-C^{*}$ is closed in the case which is of interest to us in §IV.

Definition II.7. A closed convex cone $W$ in a finite dimensional vector space $E$ is called a wedge. The vector space $H(W):=W \cap$ $(-W)$ is called the edge of $W$. We say that $W$ is pointed if $H(W)=$ $\{0\}$ and that $W$ is generating if $W=(-W)=E$, i.e., if $\operatorname{int}(W) \neq \varnothing$. A polyhedral wedge is defined to be a finite intersection of half spaces. In this terminology a closed chamber $\bar{C}$ is a generating polyhedral wedge. 
Lemma II.8. Let $X_{1}, \ldots, X_{n} \in E$. Then the convex cone

$$
W:=\sum_{i=1}^{n} \mathbb{R}^{+} X_{i}
$$

is closed, i.e., a wedge.

Proof. Set $W_{m}:=\sum_{i=1}^{m} \mathbb{R}^{+} X_{i} \subseteq W$. We prove by induction on $m$ that $W_{m}$ is closed. This is clear for $m=0,1,2$. Assume that $0 \leq m \leq n$ and that all cones which are sum of less than $m$ rays are closed. Then $W_{m}=W_{m-1}+\mathbb{R}^{+} X_{m}$. If $-\mathbb{R}^{+} X_{m} \cap W_{m-1}=\{0\}$ then the closedness of $W_{m}$ follows from [HHL89, I.2.32]. Suppose that $-X_{m} \in W_{m-1}$. Then

$$
-X_{m}=\sum_{i=1}^{m-1} \alpha_{i} X_{i}
$$

with $\alpha_{i} \geq 0$. We may assume that $\alpha_{i}>0$ iff $1 \leq i \leq k \leq m-1$. Let $F:=\operatorname{span}\left\{X_{1}, \ldots, X_{k}, X_{m}\right\}$. Since

$$
0=X_{m}+\sum_{i=1}^{k} \alpha_{i} X_{i}
$$

we conclude that $0 \in \operatorname{int}_{F}\left(\mathbb{R}^{+} X_{m}+\sum_{i=1}^{k} \mathbb{R}^{+} X_{i}\right)$ and therefore that $F=\mathbb{R}^{+} X_{m}+\sum_{i=1}^{k} \mathbb{R}^{+} X_{i}$. Let $p: E \rightarrow F^{\perp}$ denote the orthogonal projection. Then, since $F \subseteq W_{m}$,

$$
W_{m}=F+\left(W_{m} \cap F^{\perp}\right)=F+p\left(W_{m}\right)=F+\sum_{i=k+1}^{m-1} \mathbb{R}^{+} p\left(X_{i}\right) .
$$

The cone $F_{1}:=\sum_{i=k+1}^{m-1} \mathbb{R}^{+} p\left(X_{i}\right) \subseteq F^{\perp}$ is closed by the induction hypothesis. So we see that $W_{m}=F+F_{1}$ is closed because $E=$ $F \oplus F^{\perp}$.

COROllary II.9. If $C$ is a chamber, then

$$
C^{*}=\sum_{H \in \mathscr{H}} \mathbb{R}^{+} e_{H}
$$

Proof. If follows from the definition that

$$
C^{*}=\left(\bigcap_{H \in \mathscr{H}}\left(\mathbb{R}^{+} e_{H}\right)^{*}\right)^{*}=\overline{\sum_{H \in \mathscr{H}} \mathbb{R}^{+} e_{H}}
$$

In view of Lemma II. 8 this proves the assertion. 
LEMMA II.10. If $E=E_{1} \oplus E_{2}$ is an orthogonal decomposition into $\mathscr{W}$-invariant subspaces, then the following assertions hold:

(i) Every hyperplane $H \in \mathscr{H}$ decomposes as $H=\left(H \cap E_{1}\right) \oplus(H \cap$ $\left.E_{2}\right)$.

(ii) $\bar{C}=\left(\bar{C} \cap E_{1}\right)+\left(\bar{C} \cap E_{2}\right)$.

(iii) $\left.C^{*}=\left(C^{*} \cap E_{1}\right)+C^{*} \cap E_{2}\right)$.

Proof. (i) Let $s_{H}$ denote the reflection on the hyperplane $H$. Then $s_{H}$ leaves $E_{1}$ and $E_{2}$ invariant. We may assume that $\left.s_{H}\right|_{E_{1}} \neq \mathrm{id}_{E_{1}}$. Then there exists $X \in E_{1}$ such that $s_{H}(X) \neq X$. Therefore $s_{H}(X)-$ $X \in E_{1} \backslash\{0\}$ and consequently

$$
H=\left(s_{H}(X)-X\right)^{\perp}=\left(H \cap E_{1}\right) \oplus E_{2} .
$$

(ii) The set $\bar{C}$ is the intersection of half spaces bounded by hyperplanes in $\mathscr{H}$. Since all these hyperplanes are adjusted to the decomposition of $E$, the same is true for $\bar{C}$.

(iii) This follows from (ii) and $E_{1}^{\perp}=E_{1}^{*}=E_{2}$.

LEMMA II.11. Let $W \subseteq E$ be a $\mathscr{W}$-invariant wedge. Then the convex cones $W-C^{*}$ and $(W \cap \bar{C})-C^{*}$ are closed.

Proof. (i) Since $W$ is invariant, the subspace $E_{1}:=H(W)$ is also $\mathscr{W}$-invariant. Therefore $E_{2}:=E_{1}^{\perp}$ is $\mathscr{W}$-invariant and

$$
W=E_{1}+\left(W \cap E_{2}\right),
$$

where $W \cap E_{2}$ is a pointed $\mathscr{W}$-invariant wedge in $E$. We know from Lemma II.10 that

$$
C^{*}=\left(C^{*} \cap E_{1}\right)+\left(C^{*} \cap E_{2}\right)
$$

Hence

$$
W-C^{*}=H(W)+\left(\left(W \cap E_{2}\right)-\left(C^{*} \cap E_{2}\right)\right) .
$$

So it remains to show that $\left(W \cap E_{2}\right)-\left(C^{*} \cap E_{2}\right)$ is closed. Set

$$
E_{2, \text { fix }}:=\left\{X \in E_{2}:(\forall s \in \mathscr{W}) s(X)=X\right\}
$$

and

$$
E_{2, \mathrm{eff}}:=\operatorname{span}\left\{s(X)-X: X \in E_{2}, s \in \mathscr{W}\right\}
$$

Then

$$
C^{*} \cap E_{2}=\sum_{e_{H} \in E_{2} \cap E_{\mathscr{X}, c}} \mathbb{R}^{+} e_{H} \subseteq E_{2, \mathrm{eff}}
$$


and

$$
W \cap E_{2, \mathrm{eff}}=\{0\}
$$

([Ne90, I.10]). We conclude that

$$
\left(W \cap E_{2}\right) \cap\left(C^{*} \cap e_{2}\right)=\{0\} .
$$

This implies that $\left(W \cap E_{2}\right)-\left(C^{*} \cap E_{2}\right)$ is closed ([HHL89, I.2.32]).

(ii) As in (i) we have that

$$
\left(W \cap \bar{C} \cap E_{2}\right) \cap\left(C^{*} \cap E_{2}\right)=\{0\}
$$

and therefore

$$
\left(W \cap \bar{C} \cap E_{2}\right)-\left(C^{*} \cap E_{2}\right)
$$

is closed. But

$$
(W \cap \bar{C})-C^{*}=(H(W) \cap \bar{C})-\left(C^{*} \cap H(W)\right)+\left(W \cap \bar{C} \cap E_{2}\right)-\left(C^{*} \cap E_{2}\right)
$$

and so it remains to show that the cone $W^{\prime}:=(H(W) \cap \bar{C})-\left(C^{*} \cap\right.$ $H(W))$ is closed. Each of the cones $H(W) \cap \bar{C}$ and $C^{*} \cap H(W)$ is polyhedral and therefore a sum of finitely many rays ([HHL89, I.4.2]). So the same holds for $W^{\prime}$ and the closedness follows from Lemma II.8.

Proposition II.12. Let $W \subseteq E$ be a $\mathscr{W}$-invariant wedge and $X \in$ $\bar{C}$. Then

$$
\operatorname{co}(X)+W=\bigcap_{s \in \mathscr{W}} s\left(X+W-C^{*}\right)
$$

Proof. " $\subseteq$ ": First we note that $\operatorname{co}(X)+W$ is a closed convex $\mathscr{W}$ invariant set because $\operatorname{co}(X)$ is compact. Therefore it suffices to show that

$$
\operatorname{co}(X)+W \subseteq X+W-C^{*}
$$

This follows from $\operatorname{co}(X) \subseteq X-C^{*}$ (Lemma II.5).

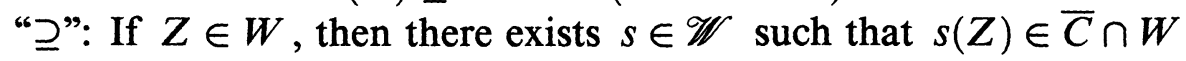
(Proposition II.2). Therefore

$$
Z=s(Z)-(s(Z)-Z) \in(W \cap \bar{C})-C^{*} .
$$

(Corollary II.3). Using Lemma II.5(iii) and Lemma II.11, we find that

$$
\begin{aligned}
\bigcap_{s \in \mathscr{W}} s\left(X+W-C^{*}\right) & =\bigcap_{s \in \mathscr{W}} s\left(X+(W \cap \bar{C})-C^{*}\right) \\
& =\operatorname{co}(X+(W \cap \bar{C})) \subseteq \operatorname{co}(X)+W .
\end{aligned}
$$


In the remainder of this section we consider the following situation: Let $E^{\prime} \subseteq E$ be a subspace, $p: E \rightarrow E^{\prime}$ the orthogonal projection,

$$
\mathscr{H}^{\prime}:=\left\{H \cap E^{\prime}: H \in \mathscr{H}, E^{\prime} \nsubseteq H\right\},
$$

and $\mathscr{W}^{\prime}$ the group generated by the reflections on the hyperplanes in $\mathscr{H}^{\prime}$. We make the following assumption:

$$
\mathscr{W}^{\prime} \subseteq\left\{\left.s\right|_{E^{\prime}}: s \in \mathscr{W}, s\left(E^{\prime}\right) \subseteq E^{\prime}\right\} .
$$

Note that $\left(\mathrm{A}^{\prime}\right)$ implies in particular that the system $\left(E^{\prime}, \mathscr{H}^{\prime}\right)$ satisfies (A1) and (A2).

LEMMA II.13. If $H=e_{H}^{\perp}$, then $H \cap E^{\prime}=p\left(E_{H}\right)^{\perp} \cap E^{\prime}$.

Proof. For $X \in E^{\prime}$ the conditions $\left\langle X, e_{H}\right\rangle=0$ and $\left\langle X, p\left(e_{H}\right)\right\rangle=0$ are equivalent. This implies the assertion.

Lemma II.14. Let $C \subseteq E$ be a chamber. Then $C^{\prime}:=\operatorname{int}_{E^{\prime}}\left(\bar{C} \cap E^{\prime}\right)$ is a chamber in $E^{\prime}, \overline{C^{\prime}}=\bar{C} \cap E^{\prime}$, and $C^{* *}=p\left(C^{*}\right)$.

Proof. Choose $e_{H} \in E$ such that $C=\left\{X \in E:(\forall H \in \mathscr{H})\left\langle X, e_{H}\right\rangle\right.$ $>0\}$. Then Lemma II.13 shows that we may set $e_{H^{\prime}}:=p\left(e_{H}\right)$ to find that

Hence

$$
\bar{C} \cap E^{\prime}=\left\{X \in E^{\prime}:\left(\forall H^{\prime} \in \mathscr{H}^{\prime}\right)\left\langle X, e_{H^{\prime}}\right\rangle \geq 0\right\} \text {. }
$$

$$
C^{\prime}=\left\{X \in E^{\prime}:\left(\forall H^{\prime} \in \mathscr{H}^{\prime}\right)\left\langle X, e_{H^{\prime}}\right\rangle>0\right\} .
$$

Therefore $C^{\prime}$ is a chamber and

$$
C^{\prime *}=\sum_{H^{\prime} \in \mathscr{H}^{\prime}} \mathbb{R}^{+} e_{H^{\prime}}=p\left(\sum_{H \in \mathscr{H}} \mathbb{R}^{+} e_{H}\right)=p\left(C^{*}\right)
$$

We note that, if $W \subseteq E$ is a wedge, then it is a general fact that

$$
\left(W \cap E^{\prime}\right)^{*}=\overline{W^{*}+E^{\prime \perp}} \cap E^{\prime}=\overline{p\left(W^{*}\right)} .
$$

For $X \in E^{\prime}$ we denote the closed convex hull of $\mathscr{W}^{\prime}(X)$ with $\operatorname{co}^{\prime}(X)$.

TheOREM II.15. Let $X \in E^{\prime}$. Then $p(\operatorname{co}(X))=\operatorname{co}^{\prime}(X)$.

Proof. Set $K:=p(\operatorname{co}(X))$. This is a compact convex subset of $E^{\prime}$. Let $s^{\prime} \in \mathscr{W}^{\prime}$ and $s \in \mathscr{W}$ with $\left.s\right|_{E^{\prime}}=s^{\prime}$. Then

$$
\begin{aligned}
s^{\prime}(K) & =s^{\prime}(p(\operatorname{co}(X)))=s(p(\operatorname{co}(X))) \\
& =p \circ s(\operatorname{co}(X))=p(\operatorname{co}(X))=K
\end{aligned}
$$


because $\operatorname{co}(X)$ is invariant under $\mathscr{W}$. Therefore $K$ is invariant under $\mathscr{W}^{\prime}$ and, since $X \in K$, we have that

$$
\operatorname{co}^{\prime}(X) \subseteq K \text {. }
$$

Choose $s^{\prime} \in \mathscr{W}^{\prime}$ such that $s^{\prime}(X) \in \overline{C^{\prime}} \subseteq \bar{C}$ and $s \in \mathscr{W}$ with $\left.s\right|_{E^{\prime}}=s^{\prime}$. We use Lemma II.14 and Lemma II.5 to obtain

$$
K=p(\operatorname{co}(X)) \subseteq p\left(s(X)-C^{*}\right)=s^{\prime}(X)-p\left(C^{*}\right)=s^{\prime}(X)-C^{\prime *} .
$$

Again with Lemma II.5 this implies that

$$
K \cap \overline{C^{\prime}} \subseteq\left(s^{\prime}(X)-C^{\prime *}\right) \cap \overline{C^{\prime}} \subseteq \operatorname{co}^{\prime}\left(s^{\prime}(X)\right)=\operatorname{co}^{\prime}(X) .
$$

Together with Proposition II. 2 and the $\mathscr{W}^{\prime}$-invariant of $K$ this completes the proof.

COROLlARY II.16. Let $W^{\prime} \subseteq E^{\prime}$ be a $\mathscr{W}^{\prime}$-invariant wedge. Then $W:=\operatorname{co}\left(W^{\prime}\right)$ is a $\mathscr{W}$-invariant wedge in $E$ with $p(W)=W \cap E^{\prime}=$ $W^{\prime}$.

Proof. Clearly $W^{\prime} \subseteq W \cap E^{\prime} \subseteq p(W)$. To see that $p(W) \subseteq W^{\prime}$ it suffices to show that $p(\mathscr{W}(X)) \subseteq W^{\prime}$ for all $X \in W^{\prime}$. This is a consequence of Theorem II.15.

REMARK II.17. Let us assume that $W_{0} \subseteq E$ is a $\mathscr{W}$-invariant edge wedge such that

$$
W_{0}^{*} \subseteq W_{0}
$$

and that $W^{\prime} \subseteq E^{\prime}$ is a $\mathscr{W}^{\prime}$-invariant wedge with

$$
p\left(W_{0}^{*}\right) \subseteq W^{\prime} \subseteq W_{0} \cap E^{\prime} .
$$

We would like to extend $W^{\prime}$ to a wedge $W$ in $E$ which is $\mathscr{W}$ invariant and which satisfies

$$
W_{0}^{*} \subseteq W \subseteq W_{0} \text { and } W \cap E^{\prime}=W^{\prime} .
$$

We set $W:=W_{0}^{*}+\operatorname{co}\left(W^{\prime}\right)$. Then $W_{0}^{*} \subseteq W \subseteq W_{0}$ and

$$
W^{\prime} \subseteq W \cap E^{\prime} \subseteq p(W) \subseteq p\left(W_{0}^{*}\right)+p\left(\operatorname{co}\left(W^{\prime}\right)\right) \subseteq W^{\prime}+W^{\prime}=W^{\prime} .
$$

III. Unitary representations with highest weight. Let $H$ be a Lie group with $\mathfrak{h}=\mathbf{L}(H), \pi: H \rightarrow U(\mathscr{H})$ a unitary representation of $H$ on the Hilbert space $\mathscr{H}, \mathscr{H}^{\infty}\left(\mathscr{H}^{\omega}\right)$ the corresponding spaces of smooth (analytic) vectors. We write $d \pi$ for the derived representation 
of $\mathfrak{h}$ on $\mathscr{H}^{\infty}$. We extend this representation to a representation of the complexified Lie algebra $\mathfrak{h}_{\mathbb{C}}$ and set

$$
W(\pi):=\{X \in i \mathfrak{h}: d \pi(X) \leq 0\} .
$$

Note that the operators $d \pi(X), X \in i$ h are essentially selfadjoint because $\mathscr{H}^{\omega}$ is dense in $\mathscr{H}$ ([We76, p. 244]). Therefore the condition $X \in W(\pi)$ is equivalent to the existence of a strongly continuous one-parameter semigroup of selfadjoint contractions on $\mathscr{H}$ whose infinitesimal generator is the closure of $d \pi(X)$.

Now let $(G, \sigma)$ be a complex symmetric Lie group, where the induced involution $\sigma$ on $\mathfrak{g}$ is complex antilinear and induces an isomorphism $\mathfrak{g} \cong \mathfrak{h}_{\mathbb{C}}$. We assume that $\mathfrak{h}$ is simple Hermitian. Note that this implies that $H=G_{0}^{\sigma}$ is real reductive in the sense of [Wal88, p. 43]. Let $\widetilde{H}$ denote the universal covering group of $H$. Then $(H \cap K)^{\sim}=\left\langle\exp _{\widetilde{H}} \mathfrak{h}_{\mathfrak{k}}\right\rangle$ because $H \cap K$ is a maximal compact subgroup of $H$.

Proposition III.1. The symmetric Lie algebra $(\mathfrak{g}, \sigma)$ has the following properties:

(i) $\operatorname{dim} \mathfrak{c}=1$.

(ii) ia is a compactly embedded Cartan subalgebra of $\mathfrak{h}$.

(iii) $\mathfrak{a}_{\mathbb{C}}$ is a Cartan subalgebra of $\mathfrak{g}$.

(iv) There exists an element $Z \in \mathfrak{c}$ such that

$$
\Delta P^{+}=\{\alpha \in \Delta: \alpha(Z)=1\} .
$$

(v) $\mathfrak{p}^{+}:=\sum_{\alpha \in \Delta_{p}^{+}} \mathfrak{g}^{\alpha}$ is an abelian subalgebra.

(vi) There exist $\operatorname{Ad}(H)$-invariant pointed cones $W_{\min } \subseteq W_{\max }$ in ih such that

$$
p\left(W_{\min }\right)=W_{\min } \cap \mathfrak{a}=C_{\min } \quad \text { and } \quad p\left(W_{\max }\right)=W_{\max } \cap \mathfrak{a}=C_{\max },
$$

where $p: i \mathfrak{h} \rightarrow \mathfrak{a}$ is the orthogonal projection.

Proof. (i) This follows immediately from $\mathfrak{c}=i Z\left(\mathfrak{h}_{\mathfrak{k}}\right)$ and $\mathfrak{q}_{\mathfrak{p}}=i \mathfrak{h}_{\mathfrak{k}}$.

(ii) Since $\mathfrak{a}$ is maximal abelian in $i \mathfrak{h}_{\mathfrak{k}}$, the subspace $i \mathfrak{a}$ is a Cartan algebra of $\mathfrak{h}_{\mathfrak{k}}$. Hence $\mathfrak{a}$ is a compactly embedded Cartan algebra of $\mathfrak{g}$ because rank $\mathfrak{h}_{\mathfrak{k}}=\operatorname{rank} \mathfrak{h}$.

(iii) This is immediate from (ii).

(iv) The existence of an element $Z \in \mathfrak{c}$ with $\overline{\operatorname{Spec}}(\operatorname{ad} Z)=\{-1,0$, $1\}$ is a consequence of [Hel78, p. 382]. Let $\alpha$ be a non-compact positive root. We normalize $Z$ such that $\alpha(Z)=1$. Suppose that 
$\beta \in \Delta_{p}^{+}$with $\beta(Z)=-1$. Then $(\alpha-\beta)(Z)=2$ and therefore $\alpha-\beta$ is no root. Moreover $(\alpha+\beta)(Z)=0$ shows that $\alpha+\beta$ cannot be a root because the compact roots are smaller than $\alpha$ (cf. Definition I.1). In the following we identify the roots with elements in $\mathfrak{a}$ via the restriction of the Cartan Killing form to $\mathfrak{a}$. If $\gamma$ is a compact root which is not orthogonal to $\beta$, then we choose the sign of $\beta$ such that $s_{\gamma}(\beta)$ is greater than $\beta$. Now $s_{\gamma}(\beta)(Z)=-1$ implies that also $\alpha \pm s_{\gamma}(\beta) \notin \Delta$. Inductively this proves that $\alpha$ is orthogonal to the Weyl group orbit $\mathscr{W} \beta$. But the span of this orbit contains $c$ because $\sum_{w \in \mathscr{W}} w(\beta)$ is $\mathscr{W}$-invariant and therefore in $\mathfrak{c}$. This is a contradiction because $\alpha(Z) \neq 0$. We conclude that $\beta(Z)=1$.

(v) If $\alpha, \beta \in \Delta_{p}^{+}$, then $(\alpha+\beta)(Z)=2$ and (iv) implies that $\alpha+\beta$ is no root. Hence $\mathfrak{p}^{+}$is abelian.

(vi) [HHL89, p. 277, 279].

We identify $\mathfrak{a}$ with its own dual via the Cartan Killing form. The corresponding coroots are denoted with $Z_{\alpha}=\frac{2 \alpha}{\langle\alpha, \alpha\rangle}$. The set $\mathscr{R}$ of highest weights of all possible irreducible unitary representations of $(H \cap K)^{\sim}$ is given by

$$
\mathscr{R}:=\left\{\lambda \in i \mathfrak{a}:\left(\forall \alpha \in \Delta_{k}^{+}\right)\left\langle\lambda, Z_{\alpha}\right\rangle \in \mathbb{N}_{0}\right\}
$$

([Wal88, p. 36]). We write $\mathscr{R}_{K}$ for the subset of $\mathscr{R}$ corresponding to the unitary representations of $H \cap K$. For $\lambda \in \mathscr{R}$ let $U_{\lambda}$ denote the corresponding irreducible representation of $\mathfrak{g}$ with highest weight $\lambda$.

If $\pi$ is an irreducible unitary representation of $H$, we denote with $\mathscr{H}_{\mathfrak{k}}$ the corresponding $\mathfrak{g}$-module of $H \cap K$-finite vectors, and set $\mathscr{H}_{\mathfrak{k}}^{0}:=$ $\left\{v \in \mathscr{H}_{\mathfrak{k}}: \mathfrak{p}^{+} . v=\{0\}\right\}$. Then $\pi$ is called a representation with highest weight if $\mathscr{H}_{\mathfrak{k}}^{0} \neq\{0\}$. In this case there exists a unique $\lambda \in \mathscr{R}$ such that the representation of $\mathfrak{g}$ in $\mathscr{H}_{\mathfrak{k}}^{0}$ is equivalent to $U_{\lambda}$ ([Wal88, p. 85], [Bou90, Ch. VIII, §6]). We write $\pi_{\lambda}$ for the corresponding irreducible unitary representation of $H$.

We write $C_{k}$ for the closed Weyl chamber in a with respect to the positive system $\Delta_{k}^{+}$of compact roots and $C_{k}^{*}$ for its dual.

Let $\mathscr{P} \subseteq \mathscr{R}_{K}$ denote the set of all functionals for which $\pi_{\lambda}$ exists. According to Theorem 3 in [HC55], which is proved in [HC56] (cf. p. 612), we know that

$$
-C_{\max } \cap \mathscr{R}_{K} \supseteq \mathscr{P} \supseteq \mathscr{R}_{1},
$$

where

$$
\mathscr{R}_{1}=\left\{\lambda \in \mathscr{R}_{K}:\left(\forall \beta \in \Delta_{p}^{+}\right)\langle\lambda+\rho, \beta\rangle<0\right\}
$$

parametrizes the holomorphic discrete series, and $\rho=\frac{1}{2} \sum_{\alpha \in \Delta^{+}} \alpha$. 
LEMMA III.2.

$$
\overline{\mathbb{R}+\mathscr{P}}=\left(-C_{\max }\right) \cap C_{k} .
$$

Proof (cf. [Ols82, 2.7]). Let $B=\left\{\alpha_{0}, \alpha_{1}, \ldots, \alpha_{n}\right\}$ be a basis of the positive system $\Delta^{+}$such that $\alpha_{0}$ is non-compact and $\left\{\alpha_{1}, \ldots, \alpha_{n}\right\}$ constitute a basis of $\Delta_{k}^{+}$. Define the element $\omega_{0} \in \mathfrak{a}^{*}$ by $\omega_{0}\left(i\left[\mathfrak{h}_{\mathfrak{k}}, \mathfrak{h}_{\mathfrak{k}}\right] \cap \mathfrak{a}\right)=\{0\}$ and $\omega_{0}(Z)=1$, where $Z \in i Z\left(\mathfrak{h}_{\mathfrak{k}}\right)$ is the element with $\beta(Z)=1$ for all $\beta \in \Delta_{p}^{+}$. Then we have that

$$
\mathscr{R}_{K}^{\prime}:=\left(\mathbb{Z} \omega_{0}+\mathbb{Z} \alpha_{1}+\cdots+\mathbb{Z} \alpha_{n}\right) \cap \mathscr{R} \subseteq \mathscr{R}_{K}
$$

([Kn86, p. 85], [Wal88, p. 88]). We set $\mathscr{R}_{1}^{\prime}:=\mathscr{R}_{K}^{\prime} \cap \mathscr{R}_{1}$. Then it suffices to show that $\overline{\mathbb{R}^{+} \mathscr{R}_{1}^{\prime}}=\left(-C_{\max }\right) \cap C_{k}$.

The inclusion

$$
\overline{\mathbb{R}^{+} \mathscr{R}_{1}^{\prime}} \subseteq \overline{\mathbb{R}^{+} \mathscr{R}_{1}} \subseteq\left(-C_{\max }\right) \cap C_{k}
$$

follows immediately from the definitions. For the converse let $\lambda \in$ $-\operatorname{int}\left(C_{\max }\right) \cap C_{k}$ and $\varepsilon>0$. We have to find an element $\nu^{\prime} \in \mathbb{R}^{+} \mathscr{R}_{1}^{\prime}$ with $\left\|\nu^{\prime}-\lambda\right\|<\varepsilon$. Then the assertion follows from the density of $-\operatorname{int}\left(C_{\max }\right) \cap C_{k}$ in $-C_{\max } \cap C_{k}$.

Since $\mathfrak{a}=\bigcup_{m \in \mathbb{N}}\left(-\operatorname{int}\left(C_{\max }\right)-m \lambda\right)$, there exists $m \in \mathbb{N}$ such that $m \lambda+\rho \in-\operatorname{int}\left(C_{\max }\right)$. Then

$$
-\delta:=\max _{\beta \in \Delta_{p}^{+}}\langle m \lambda+\rho, \beta\rangle<0
$$

holds. But $\left\{\omega_{0}, \alpha_{1}, \ldots, \alpha_{n}\right\}$ is a base of $\mathfrak{a}$, and therefore there exist $k \in \mathbb{N}$ and an element $\nu \in \mathscr{R}_{K}^{\prime}$ with

$$
\|k m \lambda-\nu\|<\min \left\{\frac{\delta}{\max _{\beta \in \Delta_{p}^{+}}\|\beta\|}, \varepsilon\right\} .
$$

For $\beta \in \Delta_{p}^{+}$this implies that

$$
\begin{aligned}
\langle\nu+\rho, \beta\rangle & =\langle\nu-k m \lambda, \beta\rangle+\langle(k-1) m \lambda, \beta\rangle+\langle m \lambda+\rho, \beta\rangle \\
& \leq\|k m \lambda-\nu\|\|\beta\|-\delta<\delta-\delta=0 .
\end{aligned}
$$

Consequently $\nu \in \mathscr{R}_{1}^{\prime}$ and the element $\nu^{\prime}:=\frac{1}{k m} \nu$ satisfies the condition

$$
\left\|\lambda-\nu^{\prime}\right\|<\frac{\varepsilon}{k m} \leq \varepsilon .
$$

Definition III.3. A pair $(S, \#)$ of a topological semigroup $S$ and an involutive antiautomorphism $s \mapsto s^{\#}$ is called an involutive semigroup. A representation of an involutive semigroup $(S, \#)$ is a homomorphism $\mathscr{T}$ from $S$ into the semigroup $\mathscr{C}(\mathscr{H})$ of all contractions 
on a complex Hilbert space $\mathscr{H}$ which is continuous with respect to the weak operator topology, and which satisfies

$$
\mathscr{T}\left(s^{\#}\right)=\mathscr{T}(s)^{*} \text {. }
$$

Theorem III.4. Let $W_{\min }$ be as in Proposition III.1. The set $S_{\min }:=$ $H \exp \left(W_{\min }\right)$ is a closed subsemigroup of $G$ and $s^{\#}:=\sigma(s)^{-1}$ defines an involutive antiautomorphism of $S$. Moreover, every representation $\pi_{\lambda}, \lambda \in \mathscr{P}$, has a continuation to a representation $\tilde{\pi}_{\lambda}$ of $\left(S_{\min }, \#\right)$ which is holomorphic on the interior $H \exp \left(\operatorname{int}\left(W_{\min }\right)\right)$ of $S_{\min }$.

Proof. [Ols82, 4.5].

Let $\lambda \in \mathscr{P}$. We write $\Delta(\lambda, \mathfrak{a})$ for the set of all weights of $\mathfrak{a}$ on $\mathscr{H}_{\mathfrak{k}}$ and $\mathscr{H}^{\mu}$ for the corresponding weight spaces, i.e.,

$$
\mathscr{H}^{\mu}=\left\{w \in \mathscr{H}_{\mathfrak{k}}:(\forall X \in \mathfrak{a}) d \pi_{\lambda}(X) w=\mu(X) w\right\} .
$$

LEMMA III.5. Every weight $\mu^{\prime} \in \Delta(\lambda, \mathfrak{a})$ which is extremal with respect an irreducible $\mathfrak{k}$-submodule is $\mathscr{W}$-conjugate to a weight of the form

$$
\mu=\lambda-\sum_{\alpha \in \Delta_{p}^{+}} n_{\alpha} \alpha,
$$

where the $n_{\alpha}$ are non-negative integers.

Proof. See for example [Sta86]. The idea is to realize the representation of $\mathfrak{g}$ on the algebra of polynomials on $\mathfrak{p}^{+}$.

LEMMA III.6. Set $S_{1}:=\exp \left(C_{\max }\right) \subseteq \exp (\mathfrak{a})$. Then there exists $a$ homomorphism $\hat{\pi}_{\lambda}: S_{1} \rightarrow \mathscr{B}(\mathscr{H})$ such that

$$
\hat{\pi}_{\lambda}\left|\exp \left(C_{\min }\right)=\tilde{\pi}_{\lambda}\right|_{\exp \left(C_{\min }\right)} .
$$

Moreover, if $X \in C_{\max } \cap C_{k}$, then

$$
\left\|\hat{\pi}_{\lambda}(\exp X)\right\|=e^{\lambda(X)} .
$$

Proof. Let $X \in C_{\max }$. Then Lemma III.5 shows that every weight $\mu \in \Delta(\lambda, \mathfrak{a})$ satisfies

$$
\mu(X) \leq \max _{s \in \mathscr{W}} \lambda(s(X))
$$

because $\mathscr{W} \Delta_{p}^{+} \subseteq \Delta_{p}^{+}$and $X \in C_{\max }$. If, in addition, $X \in C_{k}$, in view of Proposition I.2, this implies that

$$
\mu(X) \leq \lambda(X) \quad \forall \mu \in \Delta(\lambda, \mathfrak{a}) .
$$


We define

$$
\hat{\pi}_{\lambda}(\exp X)(w):=e^{\mu(X)} w \quad \forall w \in \mathscr{H}^{\mu} .
$$

Then (3.3) shows that $\hat{\pi}_{\lambda}(\exp X)$ is a bounded operator on the dense subspace $\mathscr{H}_{\mathfrak{k}}$ and therefore it permits a continuation to the whole space $\mathscr{H}$ with the same operator norm. For $X \in C_{\max } \cap C_{k}$ the relation (3.4) shows that $\left\|\hat{\pi}_{\lambda}(\exp X)\right\|=e^{\lambda(X)}$. That $\hat{\pi}_{\lambda}: S_{1} \rightarrow \mathscr{B}(\mathscr{H})$ is a semigroup homomorphism follows from the fact that

$$
\left.\hat{\pi}_{\lambda}\left(a_{1}\right) \hat{\pi}_{\lambda}\left(a_{2}\right)\right|_{\mathscr{H}_{\mathfrak{k}}}=\left.\hat{\pi}_{\lambda}\left(a_{1} a_{2}\right)\right|_{\mathscr{H}_{\mathfrak{k}}}
$$

for $a_{1}, a_{2} \in S_{1}$. It is clear that $\hat{\pi}_{\lambda}$ agrees with $\tilde{\pi}_{\lambda}$ on $\exp \left(C_{\min }\right)$ because the corresponding operators are equal on $\mathscr{H}_{\mathfrak{k}}$.

In the following we write

$\hat{\pi}_{\lambda}: \exp \left(i \mathfrak{a}+C_{\max }\right) \rightarrow \mathscr{B}(\mathscr{H}), \quad \exp (i X+Y) \mapsto \pi_{\lambda}(\exp i X) \hat{\pi}_{\lambda}(\exp Y)$ for the combination of $\hat{\pi}_{\lambda}$ with $\pi_{\lambda}$ on $i \mathfrak{a}+C_{\max }$. From Lemma III.5 it is clear that this defines a semigroup homomorphism because $\pi_{\lambda}(\exp i \mathfrak{a})$ commutes with $\hat{\pi}_{\lambda}\left(\exp C_{\max }\right)$. We recall that $\mathfrak{n}:=\bigoplus_{\alpha \in \Delta^{+}} \mathfrak{g}^{\alpha}$.

LeMmA III.7. Let $\lambda \in \mathscr{P}_{G}, X \in i \mathfrak{a}+\left(C_{\max } \cap C_{k}\right) \subseteq \mathfrak{a}_{\mathbb{C}}$, and $v \in \mathscr{H}$ a vector of highest weight $\lambda$. Then the following assertions hold:

(i) $\hat{\pi}_{\lambda}(\exp X) \cdot v=e^{\lambda(X)} v$.

(ii) $\left\|\hat{\pi}_{\lambda}(\exp X)\right\|=e^{\operatorname{Re} \lambda(X)}$.

(iii) If $s \in \operatorname{int}\left(S_{\min }\right)$ and $\lambda_{s}$ and $\rho_{s}$ denote left and right multiplication with $s$ in $S$, then

$$
d \tilde{\pi}_{\lambda}(s) d \lambda_{s}(\mathbf{1}) Z(w)=\tilde{\pi}_{\lambda}(s) d \pi_{\lambda}(Z) w
$$

and

$$
d \tilde{\pi}_{\lambda}(s) d \rho_{s}(\mathbf{1}) Z(w)=d \pi_{\lambda}(Z) \tilde{\pi}_{\lambda}(s) w
$$

for $Z \in \mathfrak{g}$ and $w \in \mathscr{H}^{\infty}$.

Proof. (i) The curve $\gamma: t \mapsto \hat{\pi}_{\lambda}(\exp (t X)) . v$ is the unique solution of the initial value problem

$$
\gamma(0)=v, \quad \gamma^{\prime}(t)=d \pi_{\lambda}(X) \gamma(t) .
$$

Since

$$
\begin{aligned}
\gamma^{\prime}(t) & =d \pi_{\lambda}(X) \hat{\pi}_{\lambda}(\exp t X) v=\hat{\pi}_{\lambda}(\exp t X) d \pi_{\lambda}(X) v \\
& =\lambda(X) \hat{\pi}_{\lambda}(\exp t X) v=\lambda(X) \gamma(t),
\end{aligned}
$$

it follows that $\gamma(t)=e^{t \lambda(X)} v$. 
(ii) Let $X=X_{1}+X_{2}$ with $X_{1} \in i \mathfrak{a}$ and $X_{2} \in C_{\max } \cap C_{k}$. Then we use Lemma II.6 to obtain that

$$
\begin{aligned}
\left\|\hat{\pi}_{\lambda}\left(\exp \left(X_{1}+X_{2}\right)\right)\right\| & =\left\|\pi_{\lambda}\left(\exp X_{1}\right) \hat{\pi}_{\lambda}\left(\exp X_{2}\right)\right\| \\
& =\left\|\hat{\pi}_{\lambda}\left(\exp X_{2}\right)\right\|=e^{\lambda\left(X_{2}\right)}=e^{\operatorname{Re} \lambda\left(X_{1}+X_{2}\right)} .
\end{aligned}
$$

(iii) First we assume that $Z \in \mathfrak{h}$. Then

$$
\tilde{\pi}_{\lambda}(s \exp (Z))=\tilde{\pi}_{\lambda}(s) \pi_{\lambda}(\exp Z)
$$

and therefore

$$
d \tilde{\pi}_{\lambda}(s) d \lambda_{s}(\mathbf{1}) Z=\tilde{\pi}_{\lambda}(s) \circ d \pi_{\lambda}(Z)
$$

on $\mathscr{H}^{\infty}$. Now the assertion follows from the complex linearity of the mappings $d \tilde{\pi}_{\lambda}(s) d \lambda_{s}(\mathbf{1})$ and $\tilde{\pi}_{\lambda}(s) \circ d \pi_{\lambda}$ (Theorem III.4). The other formula follows similarly.

Lemma III.8. Let $v \in \mathscr{H}$ be a vector of highest weight $\lambda$. Then the mapping

$$
F_{1}: \operatorname{int}\left(C_{\max }\right) \times H \rightarrow \mathscr{H}, \quad(X, h) \mapsto \hat{\pi}_{\lambda}(\exp X) \pi_{\lambda}(h) v
$$

is analytic.

Proof. Let $X \in \operatorname{int}\left(C_{\max }\right)$ and $h \in H$. Then there exists $X^{\prime} \in$ $\operatorname{int}\left(C_{\max }\right)$ such that $X \in X^{\prime}+\operatorname{int}\left(C_{\min }\right)$. For $Y \in C_{\min }$ we have that

$$
F_{1}\left(X^{\prime}+Y, h\right)=\hat{\pi}_{\lambda}\left(\exp X^{\prime}\right) \tilde{\pi}_{\lambda}(\exp Y) \pi_{\lambda}(h) v
$$

(Lemma III.6). Hence the analyticity on the open neighborhood

$$
\left(X^{\prime}+\operatorname{int}\left(C_{\min }\right)\right) \times H
$$

of $(X, h)$ follows from the linearity and the boundedness of the linear operator $\hat{\pi}_{\lambda}\left(\exp X^{\prime}\right)$ and the analyticity of the mapping

$$
\operatorname{int}\left(C_{\min }\right) \times H \rightarrow \mathscr{H}, \quad\left(Y, h^{\prime}\right) \mapsto \tilde{\pi}_{\lambda}(\exp Y) \pi_{\lambda}\left(h^{\prime}\right) v
$$

(Theorem III.4).

Proposition III.9. Let $\Omega \subseteq \mathfrak{a}_{\mathbb{C}} \times \mathfrak{n}$ be the connected component of the set of all pairs $(X, Y)$ with $\exp (X) \exp (Y) \in \operatorname{int}\left(S_{\min }\right)$ which contains $\operatorname{int}\left(C_{\min }\right)$. Suppose that $\lambda \in \mathscr{P}_{G}$ and that $v \in \mathscr{H}$ is a vector of highest weight. Then

$$
\tilde{\pi}_{\lambda}(\exp (X) \exp (Y)) v=e^{\lambda(X)} v
$$

holds for all $(X, Y) \in \Omega$. 
Proof. First we note that $\Omega$ is an open connected submanifold of the complex manifold $\mathfrak{a}_{\mathbb{C}} \times \mathfrak{n}$. We define

$$
F: \Omega \rightarrow \mathscr{H}, \quad(X, Y) \mapsto \tilde{\pi}_{\lambda}(\exp (X) \exp (Y)) v .
$$

Then $F$ is a holomorphic function because it is the composition of the holomorphic mappings $\left.\tilde{\pi}_{\lambda}\right|_{\operatorname{int}\left(S_{\min }\right)}$ (Theorem III.4) and

$$
\Omega \rightarrow \operatorname{int}\left(S_{\min }\right), \quad(X, Y) \mapsto \exp (X) \exp (Y) .
$$

Let $\left(X_{0}, Y_{0}\right) \in \Omega(X, Y) \in \mathfrak{a}_{\mathbb{C}} \times \mathfrak{n}$, and $s:=\exp \left(X_{0}\right) \exp \left(Y_{0}\right) \in$ $\operatorname{int}\left(S_{\min }\right)$. We recall the definition of the power series $f(Z)=$ $\left(1-e^{-Z}\right) / Z$ and note that $f\left(\operatorname{ad} X_{0}\right) X=X$, and that $f\left(\operatorname{ad} Y_{0}\right) Y \in \mathfrak{n}$ is a finite sum because the Lie algebra $\mathfrak{n}$ is nilpotent. Now we use Lemma III.7 and [Hel78, p. 105] to obtain

$$
\begin{aligned}
& d F\left(X_{0}, Y_{0}\right)(X, Y) \\
& =d \tilde{\pi}_{\lambda}(s)\left(d \rho_{\exp Y_{0}}\left(\exp X_{0}\right) d \exp \left(X_{0}\right) X\right. \\
& \left.\quad \quad+d \lambda_{\exp X_{0}}\left(\exp Y_{0}\right) d \exp \left(Y_{0}\right) Y\right) v \\
& =d \tilde{\pi}_{\lambda}(s)\left(d \rho_{\exp Y_{0}}\left(\exp X_{0}\right) d \lambda_{\exp X_{0}(\mathbf{1}) X}\right. \\
& \quad \quad+d \lambda_{\exp X_{0}}\left(\exp Y_{0}\right) d \lambda_{\left.\exp Y_{0}(\mathbf{1}) f\left(\operatorname{ad} Y_{0}\right) Y\right) v} \\
& =d \tilde{\pi}_{\lambda}(s)\left(d \rho_{\exp Y_{0}}\left(\exp X_{0}\right) d \rho_{\exp X_{0}}(\mathbf{1}) X+d \lambda_{s}(\mathbf{1}) f\left(\operatorname{ad} Y_{0}\right) Y\right) v \\
& =d \tilde{\pi}_{\lambda}(s)\left(d \rho_{s}(\mathbf{1}) X+d \lambda_{s}(\mathbf{1}) f\left(a d Y_{0}\right) Y\right) v \\
& =d \pi_{\lambda}(X) \tilde{\pi}_{\lambda}(s) v+\tilde{\pi}_{\lambda}(s) d \pi_{\lambda}\left(f\left(\operatorname{ad} Y_{0}\right) Y\right) v \\
& =d \pi_{\lambda}(X) F\left(X_{0}, Y_{0}\right)
\end{aligned}
$$

because $d \pi_{\lambda}(\mathfrak{n}) v=\{0\}$.

Now let $X_{1} \in \operatorname{int}\left(C_{\min }\right), U_{1}$ be an open connected neighborhood of $X_{1}$ in $\mathfrak{a}_{\mathbb{C}}$, and $U_{2}$ an open connected neighborhood of 0 such that $U_{1} \times U_{2} \subseteq \Omega$. Then the calculation above shows that

$$
F\left(X_{2}, Y_{2}\right)=F\left(X_{2}, 0\right)=e^{\lambda\left(X_{2}\right)} v \quad \text { for }\left(X_{2}, Y_{2}\right) \in U_{1} \times U_{2} \text {. }
$$

Now the analyticity of $F$ on the connected set $\Omega$ shows that $F(X, Y)$ $=e^{\lambda(X)} v$ for all $(X, Y) \in \Omega$.

IV. Symmetric spaces of regular type. In this section we complete the proof of the Convexity Theorem (Theorem I.7). We keep the notation from $\S I$. As we have already remarked at the end of $\S I$, we may assume that $Z(G)=\{\mathbf{1}\}, H$ is connected, and that $(\mathfrak{g}, \tau)$ is an irreducible symmetric Lie algebra such that $Z\left(\mathfrak{h}^{a}\right) \cap \mathfrak{q}_{\mathfrak{p}} \neq\{0\}$, i.e., $(\mathfrak{g}, \tau)$ is irreducible of regular type (cf. [Ola91], [FHO91]). 
These assumptions imply in particular that $G \cong \operatorname{Ad}(G)$ is contained in a complex Lie group $G_{\mathbb{C}}$ with $\mathbf{L}\left(G_{\mathbb{C}}\right)=\mathfrak{g}_{\mathbb{C}}$. We set $\mathfrak{g}^{c}:=\mathfrak{h}+i \mathfrak{q}$ and $G^{c}:=\left\langle\exp ^{c}\right\rangle$. We collect some facts on the structure of symmetric Lie algebras of regular type.

Proposition IV.1. For every irreducible symmetric Lie algebra $(\mathfrak{g}, \tau)$ of regular type of the following assertions hold:

(i) $\mathfrak{g}^{c}$ is a Hermitian semisimple Lie algebra.

(ii) $\mathfrak{c}:=Z\left(\mathfrak{h}^{a}\right) \cap \mathfrak{q}_{\mathfrak{p}}$ is one-dimensional.

(iii) $Z_{\mathfrak{g}}(\mathfrak{c})=\mathfrak{h}^{a}$ and $Z_{\mathfrak{q}}(\mathfrak{c})=\mathfrak{q}_{\mathfrak{p}}$.

(iv) $\mathfrak{a}$ is maximal abelian in $\mathfrak{p}$ and $\mathfrak{q}$.

(v) A root $\alpha \in \Delta(\mathfrak{g}, \mathfrak{a})$ is compact iff $\mathfrak{g}^{\alpha} \subseteq \mathfrak{h}^{a}$ iff $\mathfrak{g}^{\alpha} \cap \mathfrak{h}^{a} \neq\{0\}$.

Proof. (i) Theorem I.20.

(ii) The Lie algebra $\mathfrak{k}^{c}:=\mathfrak{h}_{\mathfrak{k}}+i \mathfrak{q}_{\mathfrak{p}}$ is maximal compactly embedded in $\mathfrak{g}^{c}$. There are two cases. If $\mathfrak{g}^{c}$ is simple, then $Z\left(\mathfrak{k}^{c}\right)$ is onedimensional ([Hel78, p. 382]) and therefore $\mathfrak{c}=i Z\left(\mathfrak{k}^{c}\right)$ is also onedimensional. If $\mathfrak{g}^{c} \cong \mathfrak{h} \oplus \mathfrak{h}$, then $\mathfrak{g} \cong \mathfrak{h}_{\mathbb{C}}, \mathfrak{h}$ is simple Hermitian, and $\mathfrak{h}^{a} \cong \mathfrak{h}_{\mathfrak{k}}+i \mathfrak{h}_{\mathfrak{k}}$. Hence $\mathfrak{c}=i Z\left(\mathfrak{h}_{\mathfrak{k}}\right)$ is one-dimensional.

(iii) This is equivalent to $Z_{\mathfrak{g}^{c}}(i \mathfrak{c})=\mathfrak{k}^{c}$. If $\mathfrak{g}^{c}$ is simple, then this follows from [Hel78, p. 382]. If $\mathfrak{g}^{c}$ is not simple, then $\mathfrak{g} \cong \mathfrak{h}_{\mathbb{C}}$ and $Z_{\mathfrak{g}}(\mathfrak{c})=Z_{\mathfrak{h}_{\mathbb{C}}}\left(\mathfrak{c}_{\mathbb{C}}\right)=\left(\mathfrak{h}_{\mathfrak{k}}\right)_{\mathbb{C}}=\mathfrak{h}^{a}$. The second assertion follows trivially from the first one.

(iv) Since $\mathfrak{c} \subseteq \mathfrak{a}$ we have that

$$
Z_{\mathfrak{p}}(\mathfrak{a}) \subseteq Z_{\mathfrak{p}}(\mathfrak{c})=\mathfrak{p} \cap \mathfrak{h}^{a}=\mathfrak{q}_{\mathfrak{p}}
$$

and that

$$
Z_{\mathfrak{q}}(\mathfrak{a}) \subseteq Z_{\mathfrak{q}}(\mathfrak{c})=\mathfrak{q} \cap \mathfrak{h}^{a}=\mathfrak{q}_{\mathfrak{p}}
$$

This shows that $\mathfrak{a}$ is maximal abelian in $\mathfrak{p}$ and $\mathfrak{q}$.

(v) That $\alpha$ is a compact root means that $\alpha(\mathfrak{c})=\{0\}$ (cf. Definition I.1). This is equivalent to $\left[\mathfrak{c}, \mathfrak{g}^{\alpha}\right]=\{0\}$ and in view of (iii) this is equivalent to $\mathfrak{g}^{\alpha} \subseteq \mathfrak{h}^{a}$. The second assertion follows from the observation that $\mathfrak{g}^{\alpha} \cap \neq\{0\}$ implies that $\alpha(\mathfrak{c})=\{0\}$.

Note that Proposition IV.1 shows that our definition of an irreducible symmetric Lie algebra of regular type agrees with the definition in [Ola91], [HO90], [FHO91] etc.

As we have already seen, there are two different cases. In the first one $\mathfrak{g} \cong \mathfrak{h}_{\mathbb{C}}$ and in the second one $\mathfrak{g}^{c}$ is simple Hermitian. To handle these two cases simultaneously we introduce some notation. 
Definition IV.2. If $\mathfrak{h}$ is a simple Hermitian Lie algebra and $\mathfrak{g} \cong \mathfrak{h}_{\mathbb{C}}$ with $\mathfrak{q}=i \mathfrak{h}$, we set $\mathfrak{g}^{\#}:=\mathfrak{g}, \tau^{\#}:=\tau, \mathfrak{h}^{\#}:=\mathfrak{h}$ etc.

If $\mathfrak{g}^{c}$ is simple Hermitian, we set $\left(\mathfrak{g}^{\#}, \tau^{\#}\right):=\left(\mathfrak{g}_{\mathbb{C}}, \bar{\tau}\right)$, where $\bar{\tau}$ is the complex antilinear extension of $\tau$. Then $\mathfrak{h}^{\#}=\mathfrak{g}^{c}$, the complex antilinear extension $\theta^{\#}$ of $\theta$ is a Cartan involution of $\mathfrak{g}_{\mathbb{C}}$ commuting with $\bar{\tau}$, and therefore the notions of Definition I.1 are also available in this case. In particular we have that $\mathfrak{h}^{a^{\#}}=\left(\mathfrak{h}^{a}\right)_{\mathbb{C}}=\left(\mathfrak{k}^{c}\right)_{\mathbb{C}}, \mathfrak{q}_{\mathfrak{p}}^{\#}=i \mathfrak{k}^{c}$, and $\mathfrak{c}^{\#}:=Z\left(\mathfrak{h}^{a \sharp}\right) \cap i \mathfrak{q}_{\mathfrak{p}}^{\#}=i Z\left(\mathfrak{k}^{c}\right)$ is one-dimensional. We choose $\mathfrak{a}^{\#}$ as a $\tau$ invariant subspace of $i \mathfrak{k}^{c}$ which contains $\mathfrak{a}$ and write $\Delta^{\#}:=\Delta\left(\mathfrak{g}^{\#}, \mathfrak{a}^{\#}\right)$ for the corresponding root system. Note that $\mathfrak{c}^{\#} \subseteq \mathfrak{a}$.

For easier reference we collect the properties of the symmetric Lie algebra $\left(\mathfrak{g}^{\#}, \tau^{\#}\right)$. In the following we identify $\mathfrak{g}$ always with the corresponding subalgebra of $\mathfrak{g}^{\#}$.

Proposition IV.3. The symmetric Lie algebra $\left(\mathfrak{g}^{\#}, \tau^{\#}\right)$ has the following properties:

(i) $\mathfrak{h}^{\#}$ is simple Hermitian and $\mathfrak{g}^{\#} \cong \mathfrak{h}_{\mathbb{C}}^{\#}$.

(ii) $\left(\mathfrak{g}^{\#}, \tau^{\#}\right)$ is an irreducible symmetric Lie algebra of regular type.

(iii) $i \mathfrak{a}^{\#}$ is a compactly embedded Cartan algebra of $\mathfrak{h}^{\#}$.

(iv) $\mathfrak{a}_{\mathbb{C}}^{\#}$ is a Cartan algebra of $\mathfrak{g}^{\#}$.

(v) $\operatorname{dim} \mathfrak{c}^{\#}=1$ and there exists an element $Z \in \mathfrak{c}^{\#}$ such that

$$
\Delta_{p}^{\#+}=\left\{\alpha \in \Delta^{\#}: \alpha(Z)=1\right\} .
$$

(vi) $\Delta_{p}^{+}=\left\{\left.\alpha\right|_{\mathfrak{a}}: \alpha \in \Delta_{p}^{\#+}\right\}$.

Proof. (i) This follows from the definition.

(ii) This follows from the simplicity of $\mathfrak{g}^{\#}$ which is a consequence of (i) and Definition IV.2.

(iii) -(v) Proposition III.1.

(vi) Take $Z$ as in (v). Then a root is non-compact if and only if it does not vanish on $Z$. Now (v) implies (vi). $C_{\min }$.

Next we have to clarify the relations between the cones $C_{\min }^{\#}$ and

LEMMA IV.4. Let $p: \mathfrak{a}^{\#} \rightarrow \mathfrak{a}$ be the orthogonal projection. Then the relation between the cones in $\mathfrak{a}$ and $\mathfrak{a}^{\#}$ may be described as follows:

(i) The set of positive non-compact roots is invariant under the Weyl group. 
(ii) The cones $C_{\min }^{\#} \subseteq C_{\max }^{\#}$ are $\mathscr{W}^{\#}$-invariant.

(iii) $C_{\max }=C_{\max }^{\#} \cap \mathfrak{a}=p\left(C_{\max }^{\#}\right)$.

(iv) $C_{\min }=p\left(C_{\min }^{\#}\right) \subseteq C_{\max }$.

(v) The subspace $\mathfrak{a} \subseteq \mathfrak{a}^{\#}$ satisfies the condition $\left(\mathrm{A}^{\prime}\right)$ of $\S \mathrm{II}$ with respect to the system of hyperplanes defined by the compact roots.

Proof. (i) If $\alpha$ is non-compact positive and $w \in \mathscr{W}^{\#}$, then $\alpha(w . Z)$ $=\alpha(Z)=1$ (Proposition IV.3).

(ii) This is a consequence of Definition I.1, (i), and Proposition III.1.

(iii) The first equality follows from Proposition IV.3(vi) and the second one from $-\tau\left(C_{\max }\right)=C_{\max }$ ([Ne90, 1.10]).

(iv) The closedness of the polyhedral cone $p\left(C_{\min }^{\#}\right)$ (Lemma II.8) entails in view of [HHL89, p. 5] that

$$
C_{\min }=C_{\max }^{*}=\left(C_{\max }^{\#} \cap \mathfrak{a}\right)^{*}=\overline{C_{\min }^{\#}+\mathfrak{a}^{\perp}} \cap \mathfrak{a}=\overline{p\left(C_{\min }^{\#}\right)}=p\left(C_{\min }^{\#}\right) .
$$

The inclusion $p\left(C_{\min }^{\#}\right) \subseteq p\left(C_{\max }^{\#}\right)=C_{\max }$ is a consequence of (iii).

(v) (cf. [OS80, 1.3], [S84]) It follows from Proposition IV.1(iv) that the subspace $\mathfrak{a}^{\#} \cap i \mathfrak{h}_{\mathfrak{k}}$ is a maximal abelian subspace of $i Z_{\mathfrak{h}_{\mathfrak{k}}}(\mathfrak{a})$. But $\left(Z_{\mathfrak{h}_{\mathfrak{t}}}(\mathfrak{a})_{\mathbb{C}}, \bar{\tau}\right)$ is an orthogonal symmetric Lie algebra and therefore all maximal abelian subspaces in $i Z_{\mathfrak{h}_{\mathfrak{e}}}(\mathfrak{a})$ are conjugate under inner automorphisms coming from $Z_{K \cap H}(\mathfrak{a})$. So we find for every $g \in K \cap H$ with $\operatorname{Ad}(g) \mathfrak{a} \subseteq \mathfrak{a}$ an element $g^{\prime} \in Z_{K \cap H}(\mathfrak{a})$ with $\operatorname{Ad}\left(g^{\prime}\right) \operatorname{Ad}(g)\left(\mathfrak{a}^{\#} \cap i \mathfrak{h}_{\mathfrak{k}}\right)=\left(\mathfrak{a}^{\#} \cap i \mathfrak{h}_{\mathfrak{k}}\right)$. Hence $g^{\prime} g \in N_{K \cap H}\left(\mathfrak{a}^{\#}\right)$ and $\left.\operatorname{Ad}\left(g^{\prime} g\right)\right|_{\mathfrak{a}}=\left.\operatorname{Ad}(g)\right|_{\mathfrak{a}}$. So every element of $\mathscr{W}$ is induced by an element in $\mathscr{W}_{0}^{\#}:=\left\{s \in \mathscr{W}^{\#}: s(\mathfrak{a}) \subseteq \mathfrak{a}\right\}$. This means that condition $\left(\mathrm{A}^{\prime}\right)$ of Section II is satisfied by the pair $\mathfrak{a} \subseteq \mathfrak{a}^{\#}$ with respect to the corresponding systems of hyperplanes defined by the compact roots.

LEMMA IV.5. If $(G, \tau)$ is irreducible of regular type, then

$$
M_{0}=Z_{K}(\mathfrak{a})_{0} \subseteq H \text { and } \Omega=H M_{0} A N=H A N .
$$

Proof. First we note that $\mathfrak{a}$ is maximal abelian in $\mathfrak{p}$ (Proposition IV.1) so that $M=Z_{K}(\mathfrak{a})$. Moreover, $\mathfrak{m}=\mathbf{L}(M)=Z_{\mathfrak{k}}(\mathfrak{a}) \subseteq Z_{\mathfrak{k}}(\mathfrak{c})=$ $\mathfrak{h}_{\mathfrak{k}}$. Therefore $\mathfrak{m} \subseteq \mathfrak{h}_{\mathfrak{k}}$ and $M_{0}=\exp \mathfrak{m} \subseteq H$.

Proposition IV.6. If $(G, \tau)$ is irreducible of regular type, then $A_{\mathrm{adm}}=\exp \left(C_{\max }\right)$. 
Proof. Let $W^{\#} \subseteq \mathfrak{q}^{\#}$ be an $\operatorname{Ad}\left(H^{\#}\right)$-invariant cone with $W^{\#} \cap \mathfrak{a}^{\#}=$ $C_{\max }^{\#}$ (Proposition III.1) and set $W:=W^{\#} \cap \mathfrak{q}$. Then

$$
W \cap \mathfrak{a}=W^{\#} \cap \mathfrak{a}=C_{\max }^{\#} \cap \mathfrak{a}=C_{\max }
$$

by Lemma IV.4. We set $\Gamma:=\exp (W)$. Then it follows from [FHO91, 2.14 ] that this is a semigroup which satisfies

$$
\Gamma \subseteq \Omega=H A N .
$$

It follows in particular that $\exp \left(C_{\max }\right) H \subseteq \Omega$ and therefore that $\exp \left(C_{\max }\right) \subseteq A_{\text {adm }}$.

To see the converse, we use sl(2, R)-reduction (cf. [HO90]). Let $X \in \mathfrak{a} \backslash C_{\max }$. Then there exists a positive non-compact root $\alpha$ such that $\alpha(X)<0$. We consider the semigroup $S_{\Omega}=\{g \in G: g \Omega \subseteq$ $\Omega\}$ (cf. Definition I.15). We choose $X_{\alpha} \in \mathfrak{g}^{\alpha}$ such that $Z_{\alpha}:=$ $\left[X_{\alpha}, \tau X_{\alpha}\right]$ satisfies $\alpha\left(Z_{\alpha}\right)=2$. Note that $\mathfrak{g}^{\alpha} \cap \mathfrak{h}^{a}=\{0\}$ (Proposition IV.1) implies that $\theta \tau\left(X_{\alpha}\right)=-X_{\alpha}$, i.e., $\theta\left(X_{\alpha}\right)=-\tau\left(X_{\alpha}\right)$. We set $\mathfrak{g}_{\alpha}:=\operatorname{span}\left\{X_{\alpha}, \theta X_{\alpha}, Z_{\alpha}\right\}$. This is a three dimensional subalgebra isomorphic to $\operatorname{sl}(2, \mathbb{R})$ which is invariant under $\theta$ and $\tau$. We set $\mathfrak{h}_{\alpha}:=\mathbb{R}\left(X_{\alpha}+\tau X_{\alpha}\right)$ and $H_{\alpha}:=\operatorname{exph} \mathfrak{h}_{\alpha}$. Let $Z_{0}:=X-t Z_{\alpha}$, where $t=\frac{1}{2} \alpha(X)<0$. Then $\alpha\left(Z_{0}\right)=0$ and therefore $\left[Z_{0}, \mathfrak{g}_{\alpha}\right]=\{0\}$. Now the formula in Example I.19 implies that

$$
\begin{aligned}
& L\left(\exp (X) \exp \left(s\left(X_{\alpha}+\tau X_{\alpha}\right)\right)\right) \\
& \quad=L\left(\exp \left(Z_{0}\right) \exp \left(t Z_{\alpha}\right) \exp \left(s\left(X_{\alpha}+\tau X_{\alpha}\right)\right)\right) \\
& \quad=L\left(\exp \left(t Z_{\alpha}\right) \exp \left(s\left(X_{\alpha}+\tau X_{\alpha}\right)\right) \exp \left(Z_{0}\right)\right) \\
& \quad=Z_{0}+L\left(\exp \left(t Z_{\alpha}\right) \exp \left(s\left(X_{\alpha}+\tau X_{\alpha}\right)\right)\right) \\
& \quad=Z_{0}+\left(t+\frac{1}{2} \log \left(1+\left(1-e^{-4 t}\right) \sinh ^{2}(s)\right)\right) Z_{\alpha} .
\end{aligned}
$$

This analytic function has no extension to $\mathbb{R}$ (as a function of $s$ ) and therefore $\exp (X) H_{\alpha}$ cannot be contained in $\Omega$. This proves that $A_{\text {adm }}=\exp \left(C_{\max }\right)$.

Now we are prepared to give a general description of the set $A_{\text {adm }}$. We thank the referee for the suggestion and the proof of the following proposition. We resume the notation from $\S I$.

Proposition IV.7. Let $(G, \tau)$ be a semisimple symmetric Lie group,

$$
\Delta_{n}^{+}:=\left\{\alpha \in \Delta^{+}: \mathfrak{g}^{\alpha} \cap\left(\mathfrak{h}_{\mathfrak{p}}+\mathfrak{q}_{\mathfrak{k}}\right) \neq\{0\}\right\}
$$

and

$$
C_{n}:=\left\{Y \in \text { mana: }\left(\forall \alpha \in \Delta_{n}^{+}\right) \alpha(X) \geq 0\right\} .
$$


Then

$$
\log A_{\mathrm{adm}}=\bigcap_{w \in \mathscr{W}} w\left(C_{n}\right)
$$

Proof. In view of Theorem I.20 it suffices to assume that $(\mathfrak{g}, \tau)$ is irreducible. We consider the three cases of Theorem I.20.

Case (i). If $(\mathfrak{g}, \tau)$ is Riemannian, then $\mathfrak{h}_{\mathfrak{p}}+\mathfrak{q}_{\mathfrak{k}}=\{0\}$, so $\Delta_{n}^{+}=\varnothing$ and $C_{n}=\mathfrak{a}=\log A_{\mathrm{adm}}$ by Theorem I.20.

Case (ii). In this case $Z\left(\mathfrak{h}^{a}\right) \subseteq \mathfrak{h}_{\mathfrak{k}}$, and in terms of roots this means that $\Delta=\Delta_{k}$ separates the points of $\mathfrak{a}$, i.e., $\Delta$ spans $\mathfrak{a}^{*}$. Since $\mathfrak{g}$ is semisimple and $(\mathfrak{g}, \tau)$ non-Riemannian, the set $\Delta_{n}^{+}$is non-empty because the involution $\theta \tau$ preserves the root spaces $\mathfrak{g}^{\alpha}, \alpha \in \Delta$, so that

$$
\mathfrak{g}^{\alpha}=\left(\mathfrak{g}^{\alpha} \cap \mathfrak{h}^{a}\right)+\left(\mathfrak{g}^{\alpha} \cap\left(\mathfrak{h}_{\mathfrak{p}}+\mathfrak{q}_{\mathfrak{k}}\right)\right)
$$

is the eigenspace decomposition of $\mathfrak{g}^{\alpha}$ with respect to $\theta \tau$.

We claim that $\Delta_{n}^{+}$spans $\mathfrak{a}^{*}$. Suppose that this is false. Then there exists $X \in \mathfrak{a}$ with $\beta(X) \neq 0$ for all $\beta \in \Delta_{n}$. Whence

$$
X \in Z_{\mathfrak{q}_{\mathfrak{p}}}\left(\mathfrak{h}_{\mathfrak{p}}+\mathfrak{q}_{\mathfrak{k}}\right) \subseteq Z_{\mathfrak{h}^{a}}\left(\mathfrak{h}_{\mathfrak{p}}+\mathfrak{q}_{\mathfrak{k}}\right) .
$$

Since $(\mathfrak{g}, \theta \tau)$ is symmetric Lie algebra, the centralizer of $\mathfrak{h}_{\mathfrak{p}}+\mathfrak{q}_{\mathfrak{k}}$ in $\mathfrak{h}^{a}$ is a $\tau$-invariant ideal in $\mathfrak{g}$. In view of the assumption that $\mathfrak{g} \neq \mathfrak{h}^{a}$, this contradicts the assumption that $\mathfrak{g}$ is irreducible.

Now the fact that $\Delta_{n}$ spans $\mathfrak{a}^{*}$ shows that $C_{n}$ is a pointed $\mathscr{W}$ invariant cone in $\mathfrak{a}$ ([HHL89, p. 5]). By assumption, $Z\left(\mathfrak{h}^{a}\right) \cap \mathfrak{a}=\{0\}$, so $\mathscr{W}$ acts on $\mathfrak{a}$ without any non-zero fixed points. Finally Theorem I.10 in [Ne90] yields $C_{n}=\{0\}=\mathfrak{a}_{\mathrm{adm}}$ (Theorem I.20).

Case (iii). Then $\Delta_{n}^{+}=\Delta_{p}^{+}, C_{n}=C_{\max }$, and the $\mathscr{W}$-invariant of $C_{\max }$ (Lemma IV.4) yields that

$$
\bigcap_{w \in \mathscr{W}} w\left(C_{n}\right)=C_{\max }
$$

Now the assertion is a consequence of Proposition IV.6.

Proposition IV.8. Let $X \in C_{\max }$ and $a=\exp (X)$. Then the set $L(a H)$ is invariant under the Weyl group $\mathscr{W}$. Moreover, if $Y \in$ $L(a H)$, then $\operatorname{co}(Y) \subseteq L(a H)$.

Proof. Set $F:=L(a H)$. The Weyl group $\mathscr{W}$ is generated by the reflections $s_{\alpha}$, where $\alpha$ is a positive compact root contained in a fixed 
set $\Sigma$ of simple indivisible roots in $\Delta_{k}^{+}$. We claim that the line segment $\left\{Y, s_{\alpha}(Y)\right\}$ between $Y$ and $s_{\alpha}(Y)$ is contained in $F$ whenever $Y \in F$ (cf. [Hel84, p. 477]). Let $\alpha$ be a simple compact root. We consider the semisimple subalgebra $\mathfrak{g}^{(\alpha)}$ of $\mathfrak{g}$ which is generated by the root spaces $\mathfrak{g}^{\alpha}$ and $\mathfrak{g}^{-\alpha}$ ([Hel78, p. 407]). Note that $\mathfrak{g}^{(\alpha)} \subseteq \mathfrak{h}^{a}$ by Proposition IV.1. Then

$$
\mathfrak{g}^{(\alpha)}=\mathfrak{g}^{\alpha}+\mathfrak{g}^{2 \alpha}+\mathfrak{g}^{-\alpha}+\mathfrak{g}^{-2 \alpha}+\mathbb{R} X_{\alpha}+\mathfrak{m} \cap \mathfrak{g}^{(\alpha)},
$$

where $X_{\alpha}$ is the unique element in $\mathfrak{a}$ which satisfies $\left\langle X_{\alpha}, Y\right\rangle=\alpha(Y)$ for all $Y \in \mathfrak{a}$ and $\mathfrak{m}=Z_{\mathfrak{k} \cap \mathfrak{h}}(\mathfrak{a})$. Thus $\tau\left(\mathfrak{g}^{\alpha}\right)=\mathfrak{g}^{-\alpha}$ shows that $\tau\left(\mathfrak{g}^{(\alpha)}\right)=\mathfrak{g}^{(\alpha)}$. We set $(\alpha):=\Delta^{+} \cap\{\alpha, 2 \alpha\}$,

$$
\mathfrak{n}^{\prime}:=\sum_{\beta \in \Delta^{+} \backslash(\alpha)} \mathfrak{g}^{\beta}, \quad \text { and } \quad \mathfrak{n}^{(\alpha)}:=\mathfrak{g}^{\alpha}+\mathfrak{g}^{2 \alpha} .
$$

Then

$$
\mathfrak{n}=\mathfrak{n}^{\prime}+\mathfrak{n}^{(\alpha)} \quad \text { and } \quad\left[\mathfrak{g}^{(\alpha)}, \mathfrak{n}^{\prime}\right] \subseteq \mathfrak{n}^{\prime}
$$

because $\alpha$ is simple and therefore $s_{\alpha}\left(\Delta^{+} \backslash(\alpha)\right) \subseteq \Delta^{+}$. According to [Hel84, pp. 440, 477] we have the diffeomorphic decomposition $N=$ $N^{\alpha} N^{\prime}$, where $N^{\prime}=\exp \mathfrak{n}^{\prime}$ and $N^{\alpha}=\exp \mathfrak{n}^{(\alpha)}$.

Let $Y \in F$ and $b=\exp (Y)$. Then there exist $h, v \in H$ and $n \in N$ such that

$$
a v=h b n \text {. }
$$

We decompose $Y=Y_{\alpha}+Y_{\alpha}^{\perp}$, where $Y_{\alpha} \in \mathbb{R} X_{\alpha}$ and $Y_{\alpha}^{\perp} \in X_{\alpha}^{\perp}$. Then

$$
s_{\alpha}(Y)=s_{\alpha}\left(Y_{\alpha}\right)+Y_{\alpha}^{\perp}=-Y_{\alpha}+Y_{\alpha}^{\perp}
$$

and

$$
\left\{Y, s_{\alpha}(Y)\right\}=[-1,1] Y_{\alpha}+Y_{\alpha}^{\perp} .
$$

We put $b_{\alpha}:=\exp \left(Y_{\alpha}\right), b_{\alpha}^{\perp}:=\exp Y_{\alpha}^{\perp}$ and write $n=n_{\alpha} n^{\prime}$ in accordance with $N=N^{\alpha} N^{\prime}$. Then

$$
h^{-1} a v=b n=b_{\alpha} b_{\alpha}^{\perp} n_{\alpha} n^{\prime}=b_{\alpha} n_{\alpha} b_{\alpha}^{\perp} n^{\prime} .
$$

Let $c_{\alpha} \in \exp \left([-1,1] Y_{\alpha}\right)$ and set $G^{\alpha}:=\left\langle\exp \mathfrak{g}^{(\alpha)}\right\rangle$. Then $G^{\alpha} \subseteq H^{a}=$ $(H \cap K) \exp \left(\mathfrak{q}_{\mathfrak{p}}\right)$. According to Lemma 10.7 in [Hel84, p. 476] there exist elements $k_{\alpha}, v_{\alpha} \in G^{\alpha} \cap K \subseteq H$ and $n_{\alpha}^{0} \in N^{\alpha}$ such that

$$
k_{\alpha} b_{\alpha} n_{\alpha} v_{\alpha}=c_{\alpha} n_{\alpha}^{0} \text {, }
$$

whence $\left[X_{\alpha}^{\perp} \cap \mathfrak{a}, \mathfrak{g}^{(\alpha)}\right]=\{0\}$ and (4.2) imply that

$$
k_{\alpha} h^{-1} a v v_{\alpha}=c_{\alpha} n_{\alpha}^{0} v_{\alpha}^{-1} b_{\alpha}^{\perp} n^{\prime} v_{\alpha}=c_{\alpha} b_{\alpha}^{\perp} n_{\alpha}^{0} v_{\alpha}^{-1} n^{\prime} v_{\alpha} \text {. }
$$


We use (4.1) to see that

$$
n_{\alpha}^{0} v_{\alpha}^{-1} n^{\prime} v_{\alpha} \in n_{\alpha}^{0} N^{\prime} \subseteq N
$$

Thus

$$
L\left(k_{\alpha} h^{-1} a v v_{\alpha}\right)=L\left(a v v_{\alpha}\right)=\log \left(c_{\alpha} b_{\alpha}^{\perp}\right)=\log c_{\alpha}+Y_{\alpha}^{\perp} .
$$

Since $c_{\alpha}$ was arbitrary in $\exp \left([-1,1] Y_{\alpha}\right)$ we conclude that

$$
\left\{Y, s_{\alpha}(Y)\right\} \subseteq L(a H) \text {. }
$$

This proves the $\mathscr{S}$-invariance of $L(a H)$ because $\mathscr{W}$ is generated by the reflections $s_{\alpha}$ for $\alpha$ simple. Let $\beta$ be an arbitrary indivisible compact root. Then there exists $w \in \mathscr{W}$ such that $w . \beta$ is simple. Then we have for each $Y \in F$ that

$$
w\left\{w^{-1} Y, s_{\alpha} w^{-1} . Y\right\}=\left\{Y, w s_{\alpha}^{-1} w^{-1} . Y\right\}=\left\{Y, s_{w . \alpha} . Y\right\} \subseteq F .
$$

Now Lemma 10.4 in [Hel84, p. 474] implies that $\operatorname{co}(Y) \subseteq F$ for every element $Y \in F$.

LemMA IV.9. Let $X=\log a \in C_{\max }$ and $\alpha \in \Delta_{p}^{+}$such that $\alpha(X)>$ 0 . Then

$$
X+\mathbb{R}^{+} Z_{\alpha} \subseteq L(a H),
$$

where $\alpha\left(Z_{\alpha}\right)=2$ and $Z_{\alpha}=\left[X_{\alpha}, \tau X_{\alpha}\right]$ for $X_{\alpha} \in \mathfrak{g}^{\alpha}$.

Proof. We proceed as in the proof of Proposition IV.6 and we use the same notation. We also set $Z_{0}:=X-t Z_{\alpha}$, where $t=\frac{1}{2} \alpha(X)>0$. Then $\alpha\left(Z_{0}\right)=0$ and $\left[Z_{0}, \mathfrak{g}_{\alpha}\right]=\{0\}$. Again the formula in Example I.19 implies that

$$
\begin{aligned}
& L\left(\exp (X) \exp \left(s\left(X_{\alpha}+\tau X_{\alpha}\right)\right)\right) \\
& \quad=Z_{0}+\left(t+\frac{1}{2} \log \left(1+\left(1-e^{-4 t}\right) \sinh ^{2}(s)\right)\right) Z_{\alpha} \\
& \quad=X+\frac{1}{2} \log \left(1+\left(1-e^{-4 t}\right) \sinh ^{2} s\right) Z_{\alpha}
\end{aligned}
$$

Hence

$$
L\left(\exp (X) \exp \left(\mathbb{R}\left(X_{\alpha}+\tau X_{\alpha}\right)\right)\right)=X+\mathbb{R}^{+} Z_{\alpha}
$$

DEFINITION IV.10. We write

$$
C_{k}:=\left\{X \in \mathfrak{a}:\left(\forall \alpha \in \Delta_{k}^{+}\right) \alpha(X) \geq 0\right\}
$$

for the closed Weyl chamber with respect to the positive compact roots. Note that $C_{k}^{*}=\sum_{\alpha \in \Delta_{k}^{+}} \mathbb{R}^{+} Z_{\alpha}$, where $Z_{\alpha} \in \mathfrak{a}$ is defined by $Z_{\alpha}^{\perp}=\operatorname{ker} \alpha$ and $\alpha\left(Z_{\alpha}\right)=2$. If we identify $\mathfrak{a}$ with $\mathfrak{a}^{*}$ via $\langle X, Y\rangle:=$ $B(X, Y)$, where $B$ is the Cartan Killing form of $\mathfrak{g}$, then

$$
Z_{\alpha}=\frac{2}{\langle\alpha, \alpha\rangle} \alpha .
$$


LEMMA IV.11. There exists a non-compact root $\gamma \in \Delta_{p}^{+}$such that

$$
C_{\min } \subseteq \mathbb{R}^{+} Z_{\gamma}-C_{k}^{*} \text { and } C_{\min }=\sum_{s \in \mathscr{W}} \mathbb{R}^{+} s\left(Z_{\gamma}\right) .
$$

Proof. Let $\Sigma=\left\{\alpha_{0}, \alpha_{1}, \ldots, \alpha_{l}\right\}$ be a basis of the positive system $\Delta^{+}$([Hel78, p. 531]). Set $\Sigma_{k}:=\Sigma \cap \Delta_{k}^{+}$. Every element $\alpha \in \Sigma \backslash \Sigma_{0}$ satisfies $\alpha(Z)=1$ (Proposition IV.3). Let $\lambda=\sum_{\beta \in \Sigma} n_{\beta} \beta \in \Delta_{k}^{+}$with $n_{\beta} \in \mathbb{N}_{0}$. Then $\lambda(Z)=0$ and therefore $n_{\beta}=0$ whenever $\beta \in \Delta_{p}^{+}$. The system $\Delta_{k}$ is the system of restricted roots of the Lie algebra $\mathfrak{h}^{a}$. Therefore

$$
|\Sigma|-\left|\Sigma_{0}\right|=\operatorname{dim} \mathfrak{c}=1
$$

(Proposition IV.3). Hence $\Sigma$ contains exactly one non-compact root and we may assume that $\alpha_{0}$ is non-compact. Let $\Delta_{1} \subseteq \Delta$ be the irreducible subsystem which contains $\alpha_{0}$ and therefore all non-compact roots, and write $\gamma=\sum_{i=0}^{l} n_{i} \alpha_{i}$ for the highest root in $\Delta_{1}$ ([Bou81, Ch. VI, $\S 1$, no. 1.8]). Then $\gamma$ is non-compact because $n_{0}=1$ and every non-compact root $\beta=\sum_{i=0}^{l} m_{i} \alpha_{i} \in \Delta_{p}^{+}$satisfies $m_{i} \leq n_{i}$ for $i \geq 1$ and $m_{0}=1$. Hence $\beta=\gamma-\sum_{i=1}^{l}\left(n_{i}-m_{i}\right) \alpha_{i}$ and consequently

$$
\begin{gathered}
Z_{\beta}=\frac{2}{\langle\beta, \beta\rangle} \beta \in \mathbb{R}^{+}\left(\gamma-\sum_{i=1}^{l}\left(n_{i}-m_{i}\right) \alpha_{i}\right) \\
\subseteq \mathbb{R}^{+} Z_{\gamma}-\sum_{i=1}^{l} \mathbb{R}^{+} Z_{\alpha_{i}}=\mathbb{R}^{+} Z_{\gamma}-C_{k}^{*} .
\end{gathered}
$$

Now we apply Lemma II.5(iv) to find that

$$
\operatorname{co}\left(\mathbb{R}^{+} Z_{\gamma}\right) \supseteq \bigcap_{s \in \mathscr{W}} s\left(\mathbb{R}^{+} Z_{\gamma}-C_{k}^{*}\right) \supseteq \bigcap_{s \in \mathscr{W}} s\left(C_{\min }\right)=C_{\text {min }}
$$

(Lemma IV.4). Thus

$$
C_{\min }=\operatorname{co}\left(\mathbb{R}^{+} X_{\gamma}\right)=\sum_{s \in \mathscr{W}} \mathbb{R}^{+} s\left(Z_{\gamma}\right) .
$$

Lemma IV.12. Let $X \in C_{\max }$ and $w \in \mathscr{W}$. Then

$$
L(\exp (X) H)=L(\exp (w \cdot X) H) .
$$

Proof. We choose $g \in N_{H \cap K}(\mathfrak{a})$ such that $\left.\operatorname{Ad}(g)\right|_{\mathfrak{a}}=w$ and set $a:=\exp X$. Then $\exp w(X)=g a g^{-1}$ and therefore

$$
\begin{aligned}
L(a H) & =L\left(a g^{-1} H\right)=L\left(g^{-1} g a g^{-1} H\right) \\
& =L\left(g a g^{-1} H\right)=L(\exp (w \cdot X) H) .
\end{aligned}
$$


Proposition IV.13. Let $a \in \exp \left(C_{\max }\right) \backslash\{1\}$. Then

$$
\operatorname{co}(\log a)+C_{\min } \subseteq L(a H)
$$

Proof. In view of Lemma IV.12 and Proposition I.2 we may assume that $X \in C_{k} \backslash\{0\}$. Since $X \neq 0$, there exists a non-compact positive root $\beta$ such that $\beta(X)>0$. Let $\gamma$ denote the highest non-compact root (Lemma IV.11). Then $\gamma(X) \geq \beta(X)>0$ because $X \in C_{k}$. Using Lemma IV.9 we see that

$$
X+\mathbb{R}^{+} Z_{\gamma} \subseteq L(a H) .
$$

In view of Proposition IV.8 it remains to be proved that

$$
\operatorname{co}\left(X+\mathbb{R}^{+} Z_{\gamma}\right)=\operatorname{co}(X)+C_{\min } .
$$

Since $\mathbb{R}^{+} Z_{\gamma} \subseteq C_{\min }$ and both sets are closed, convex and invariant under $\mathscr{W}$, it suffices to show that

$$
\left(\operatorname{co}(X)+C_{\min }\right) \cap C_{k} \subseteq \operatorname{co}\left(X+\mathbb{R}^{+} Z_{\gamma}\right) \cap C_{k} .
$$

But Lemma IV.11 and Lemma I.5 imply that

$$
\operatorname{co}(X)+C_{\min } \subseteq X-C_{k}^{*}+\mathbb{R}^{+} Z_{\gamma}-C_{k}^{*}=X+\mathbb{R}^{+} Z_{\gamma}-C_{k}^{*} .
$$

Again with Lemma I.5 this leads to

$$
\begin{aligned}
\left.\left(\operatorname{co}(X)+C_{\min }\right) \cap C_{k}\right) & \subseteq\left(X+\mathbb{R}^{+} Z_{\gamma}-C_{k}^{*}\right) \cap C_{k} \\
& \subseteq \operatorname{co}\left(X+\mathbb{R}^{+} Z_{\gamma}\right) \cap C_{k} .
\end{aligned}
$$

LEMMA IV.14. Let $(\mathfrak{g}, \tau)$ be an irreducible symmetric Lie algebra of regular type. Then

$$
\begin{aligned}
C(a) & =\left\{X \in C_{\min }:\left(\forall \alpha \in \Delta_{p}^{+}, \alpha(\mathscr{W} \log a)=\{0\}\right) \alpha(Y) \leq 0\right\} \\
& = \begin{cases}C_{\min } & \text { if } a \neq \mathbf{1}, \\
\{0\} & \text { if } a=\mathbf{1} .\end{cases}
\end{aligned}
$$

Proof. If $a=1$, then $C(a)=C_{\min } \cap-C_{\max }=\{0\}$ because $C_{\max }$ is pointed and $C_{\min } \subseteq C_{\max }$ (Proposition III.1, Lemma IV.4).

If $a \neq 1$, then

$$
0 \neq \sum_{w \in \mathscr{W}} w(\log a) \in \mathfrak{c} \cap C_{\max }
$$

Hence $\alpha(Y)=0$ is equivalent to $\alpha \in \Delta_{k}$, so $C(a)=C_{\min }$. 
We note that, in view of $A_{\mathrm{adm}}=\exp \left(C_{\mathrm{max}}\right)$ and

$$
C(a)=C_{\min } \quad \forall a \in A_{\mathrm{adm}} \backslash\{\mathbf{1}\},
$$

Proposition IV.13 proves the inclusion

$$
\operatorname{co}(\log a)+C(a) \subseteq L(a H)
$$

of the Convexity Theorem.

Proposition IV.15. Let $X \in C_{\max } \cap C_{k}$ and $a=\exp (X)$. Then

$$
L(a H)-\log (a) \subseteq C_{\min }-C_{k}^{*} .
$$

Proof. We recall that we assume that $H$ is connected. By continuity and closedness of the cone $C_{\min }-C_{k}^{*}$ (Lemma II.11) we may assume that $X \in \operatorname{int}\left(-C_{\max }\right) \cap C_{k}$ because $\operatorname{int}\left(C_{\max } \cap C_{k}\right) \subseteq \operatorname{int}\left(C_{\max }\right) \cap C_{k}$ is dense in the generating cone $C_{\max } \cap C_{k}$.

Recall the definition of the complex symmetric Lie group $\left(G^{\#}, \tau^{\#}\right)$, where $H^{\#}:=\left\langle\exp \mathfrak{h}^{\#}\right\rangle$ is a simple Hermitian Lie group and $G^{\#} \cong H_{\mathbb{C}}^{\#}$.

Let $\lambda \in \mathscr{P}_{H^{*}}$. Then, according to Theorem III.4, the representation $\pi_{\lambda}: H^{\#} \rightarrow \mathscr{U}(\mathscr{H})$ has a holomorphic extension to a representation $\tilde{\pi}_{\lambda}: S_{\min }=H^{\#} \exp \left(W_{\text {min }}^{\#}\right) \rightarrow \mathscr{C}(\mathscr{H})$. Let $v \in \mathscr{H}$ be a vector of highest weight with $\|v\|=1$.

We define the analytic mappings $\alpha: H^{\#} A^{\#} N^{\#} \rightarrow H^{\#}$ and $\beta: H^{\#} A^{\#} N^{\#}$ $\rightarrow N^{\#}$ (cf. $\left.§ \mathrm{I}\right)$ by

$$
g=\alpha(g) \exp (L(g)) \beta(g) \quad \forall g \in H^{\#} A^{\#} N^{\#} .
$$

Let

$$
F_{1}: \operatorname{int}\left(C_{\max }\right) \times H^{\#} \rightarrow \mathscr{H}, \quad(X, h) \mapsto \hat{\pi}_{\lambda}(\exp X) \pi_{\lambda}(h) v
$$

be the analytic mapping from Lemma III.8. We define another mapping

$$
F_{2}: \operatorname{int}\left(C_{\max }\right) \times H^{\#} \rightarrow \mathscr{H}, \quad(X, h) \mapsto \pi_{\lambda}(\alpha(\exp (X) h)) e^{\lambda(L(\exp (X) h))} v
$$

This mapping is well defined because $\exp \left(C_{\max }\right)=A_{\mathrm{adm}}$ (Proposition IV.6) and it is analytic because $Y \mapsto \hat{\pi}_{\lambda}(\exp Y) v$ is analytic on $\operatorname{int}\left(C_{\max }\right)$ (Lemma III.8). We claim that $F_{1}=F_{2}$. Since both are analytic and $\operatorname{int}\left(C_{\max }\right) \times H^{\#}$ is connected, it suffices to show that they are equal on $\operatorname{int}\left(C_{\min }\right) \times H^{\#}$. So let $X_{0} \in \operatorname{int}\left(C_{\min }\right), a_{0}:=\exp \left(X_{0}\right)$, and

$$
\gamma(h):=\left(L\left(a_{0} h\right), \log \beta\left(a_{0} h\right)\right) \in \mathfrak{a} \times \mathfrak{n} \subseteq \mathfrak{a}_{\mathbb{C}}^{\#} \times \mathfrak{n}^{\#} .
$$

Then, since $H$ was supposed to be connected, $\gamma(H)$ is contained in the connected component of the set of all pairs $(X, Y) \in \mathfrak{a}_{\mathbb{C}}^{\#} \times \mathfrak{n}^{\#}$ 
for which $\exp (X) \exp (Y) \in \operatorname{int}\left(S_{\min }\right)$ because $\exp \left(L\left(a_{0} h\right)\right) \beta\left(a_{0} h\right)=$ $\alpha\left(a_{0} h\right)^{-1} a_{0} y \in \operatorname{int}\left(S_{\min }\right)$. Thus Proposition III.9 shows that

$$
\tilde{\pi}_{\lambda}\left(\exp \left(L\left(a_{0} h\right)\right) \beta\left(a_{0} h\right)\right) v=e^{\lambda\left(L\left(a_{0} h\right)\right)} v .
$$

We conclude that

$$
\begin{aligned}
F_{2}\left(X_{0}, h\right) & =\pi_{\lambda}\left(\alpha\left(a_{0} h\right)\right) e^{\lambda\left(L\left(a_{0} h\right)\right)} v \\
& =\pi_{\lambda}\left(\alpha\left(a_{0} h\right)\right) \tilde{\pi}_{\lambda}\left(\exp \left(L\left(a_{0} h\right)\right) \beta\left(a_{0} h\right)\right) v \\
& =\tilde{\pi}_{\lambda}\left(\alpha\left(a_{0} h\right) \exp \left(L\left(a_{0} h\right)\right) \beta\left(a_{0} h\right)\right) v \\
& =\tilde{\pi}_{\lambda}\left(a_{0} h\right) v=\tilde{\pi}_{\lambda}\left(a_{0}\right) \pi_{\lambda}(h) v \\
& =\hat{\pi}_{\lambda}\left(a_{0}\right) \pi_{\lambda}(h) v=F_{1}\left(X_{0}, h\right)
\end{aligned}
$$

for all $X_{0} \in \operatorname{int}\left(C_{\min }\right)$ and $h \in H^{\#}$. Hence $F_{1}=F_{2}$. Taking norms we obtain with Lemma III.7 that

$$
\begin{aligned}
\left\|F_{2}(X, h)\right\| & =\left\|\pi_{\lambda}(\alpha(a h)) e^{\lambda(L(a h))} v\right\| \\
& =e^{\lambda(L(a h))}\left\|\pi_{\lambda}(\alpha(a h)) v\right\| \\
& =e^{\lambda(L(a h))}=\left\|F_{1}(X, h)\right\|=\left\|\hat{\pi}_{\lambda}(a) \pi_{\lambda}(h) v\right\| \\
& \leq\left\|\hat{\pi}_{\lambda}(a)\right\|\left\|\pi_{\lambda}(h) v\right\|=\left\|\hat{\pi}_{\lambda}(a)\right\|=e^{\lambda(X)} .
\end{aligned}
$$

We conclude that

$$
\lambda(L(a h)-\log (a)) \leq 0
$$

for all $\lambda \in \mathscr{P}_{H^{*}}$, i.e.,

$$
\begin{aligned}
\log (a)-L(a h) & \in\left(\left(-C_{\max }^{\#}\right) \cap C_{k}^{\#}\right)^{*} \cap \mathfrak{a} \\
& \subseteq\left(\left(-C_{\max }\right) \cap C_{k}\right)^{*} \\
& =\frac{-C_{\min }+C_{k}^{*}}{=}=-C_{\min }+C_{k}^{*}
\end{aligned}
$$

because the cone $C_{\min }-C_{k}^{*}$ is closed (Lemma II.11). Finally this proves that

$$
L(a H) \subseteq \log (a)+C_{\min }-C_{k}^{*}
$$

THEOREM IV.16 (The Convexity Theorem for groups of regular type). Let $(G, \tau)$ be an irreducible symmetric group of regular type. Then $A_{\mathrm{adm}}=\exp \left(C_{\max }\right)$ and for $X \in C_{\max } \backslash\{0\}$ we have that

$$
L(\exp (X) H)=\operatorname{co}(X)+C_{\min } \text {. }
$$

Proof. Propositions I.12, IV.6, IV.7, IV.13, IV.15. 
REMARK IV.17. With the notations of Theorem IV.16 and $0 \neq X \in$ $-C_{\max }$ we have that

$$
\bar{L}(H \exp (X))=\operatorname{co}(X)-C_{\min },
$$

where $\bar{L}: N A H \rightarrow \mathfrak{a}, n a h \mapsto \log a$.

Proof. Set $X^{\prime}:=-X$. Then the assertion follows from Theorem IV.16 and from $\bar{L}(g)=-L\left(g^{-1}\right)$.

Corollary IV.18. Let $X \in \mathbb{R}^{+} Z \backslash\{0\}$. Then

$$
L(\exp (X) H)=X+C_{\min } .
$$

Proof. Theorem IV.16 and the invariance of $X$ under the Weyl group.

\section{Applications of the Convexity Theorem.}

Proposition IV.19. Let $a \in \exp \left(-C_{\max }\right)$ and $\bar{n} \in \bar{N} \cap H A N$. Then $a^{-1} \bar{n} a \in H A N$ and

$$
L\left(a^{-1} \bar{n} a\right)-L(\bar{n}) \in C_{\min }
$$

Proof. Let $\alpha: H A N \rightarrow H$ denote the projection. Then $L(x y)=$ $L(x \alpha(y))+L(y)$ implies that

$$
L\left(a^{-1} \bar{n} a\right)=L\left(a^{-1} \alpha(\bar{n})\right)+L(\bar{n})+\log a .
$$

Now the Convexity Theorem shows that

$$
L\left(a^{-1} \alpha(\bar{n})\right) \in L\left(a^{-1} H\right) \subseteq-\log a+C_{\min } .
$$

and the assertion follows.

Corollary IV.20. $L(\bar{N} \cap H A N) \subseteq-C_{\min }$.

Proof. Let $X \in \operatorname{int}\left(-C_{\max } \cap-C_{k}\right)$ and $\bar{n} \in \bar{N} \cap H A N$. Then

$$
\lim _{t \rightarrow \infty} \exp (-t X) \bar{n} \exp (t X)=\{1\}
$$

and therefore

$$
\lim _{t \rightarrow \infty} L(\exp (-t X) \bar{n} \exp (t X))-L(\bar{n})=-L(\bar{n}) \in C_{\min } .
$$




\section{REFERENCES}

[vdB86] E. van den Ban, $A$ convexity theorem for semisimple symmetric spaces, Pacific J. Math., 124 (1986), 21-55.

[Bou81] N. Bourbaki, Groupes et algèbres de Lie, Chapitres 4, 5 et 6, Masson, Paris, 1981.

[Bou90] _ Groupes et algèbres de Lie, Chapitres 7 et 8, Masson, Paris, 1990.

[FHO91] J. Faraut, J. Hilgert, and G. Olafsson, Analysis on ordered symmetric spaces, in preparation.

[HC55] Harish-Chandra, Representations of semi-simple Lie groups, IV, Amer. J. Math., 77 (1955), 743-777.

[HC56] _ Representations of semi-simple Lie groups, VI, Amer. J. Math., 78 (1956), 564-628.

[Hel78] S. Helgason, Differential Geometry, Lie Groups, and Symmetric Spaces, Academic Press, London, 1978.

[Hel84] _ _ Groups and Geometric Analysis, Academic Press, London, 1984.

[Hi89] J. Hilgert, Invariant Cones in Symmetric Spaces of Hermitean type, unpublished note.

[HHL89] J. Hilgert, K. H. Hofmann and J. D. Lawson, Lie Groups, Convex Cones, and Semigroups, Oxford University Press, 1989.

[HO90] J. Hilgert and G. Olafsson, Causal symmetric spaces, in preparation.

[Kn86] A. W. Knapp, Representation Theory of Semisimple Lie Groups, Princeton University Press, Princeton, New Jersey, 1986.

[Ko73] B. Kostant, On convexity, the Weyl group and the Iwasawa decomposition, Ann. Sci. Ecole Norm. Sup., 6 (1973), 413-455.

[Lo69] O. Loos, Symmetric Spaces I: General Theory, Benjamin, New York, Amsterdam, 1969.

[Ne90] K.-H. Neeb, Invariant subsemigroups of Lie groups, Mem. Amer. Math. Soc., to appear.

[Ne91a] _ Conal orders on homogeneous spaces, Invent. Math., 104 (1991), 467-496.

[Ne91b] _ Monotone functions on symmetric spaces, Math. Ann., 291 (1991), 261-273.

[Ola87] G. Olafsson, Fourier and Poisson Transformation associated to a semisimple symmetric space, Invent. Math., 90 (1987), 605-629.

[Ola90] _ Causal symmetric spaces, Math. Gottingensis, 15, preprint, 1990.

[Ola91] _ Symmetric spaces of Hermitean type, Differential Geom. Appl., 1 (1991), 195-233.

[Ols82] G. I. Ol'shanskiǐ, Invariant cones in Lie algebras, Lie semigroups, and the holomorphic discrete series, Funct. Anal. Appl., 15 (1982), 275-285.

[OS80] T. Oshima and J. Sekiguchi, Eigenspaces of invariant differential operators on affine symmetric spaces, Invent. Math., 57 (1980), 1-81.

[Ru88] W. A. F. Ruppert, On open subsemigroups of connected groups, Semigroup Forum, 39 (1988), 347-362.

[S84] H. Schlichtkrull, Hyperfunctions and Harmonic Analysis on Symmetric Spaces, Progress in Math., vol. 84, Birkhäuser, 1984.

[Sta76] R. J. Stanton, Analytic extension of the holomorphic discrete series, Amer. J. Math., 108 (1986), 1411-1424.

[Wal88] N. R. Wallach, Real Reductive Groups I, Academic Press., Boston, New York, Tokyo, 1988. 
[Wa72] G. Warner, Harmonic Analysis on Semisimple Lie Groups I, Springer, Berlin, Heidelberg, New York, 1972.

[We76] J. Weidmann, Lineare Operatoren in Hilberträumen, Teubner, Stuttgart, 1976.

Received October 7, 1991 and in revised form July 22, 1992.

FACHBEREICH MATHEMATIK

ARBEITSGRUPPE 5

TeChNische Hochschule DarmstadT

SCHLOSSGARTENSTR. 7

W-6100 DARMSTADT

RFA

AND

UNIVERSITÉ PARIS VI

ANAlyse Complexe et Géometrie

4, PLACE JUSSIEU

75252 Paris, Cedex 05

FRANCE 



\title{
PACIFIC JOURNAL OF MATHEMATICS
}

\author{
Founded by
}

\author{
E. F. BECKENBACH (1906-1982) F. Wolf (1904-1989)
}

\section{EDITORS}

Sun-Yung A. Chang

(Managing Editor)

University of California

Los Angeles, CA 90024-1555

chang@math.ucla.edu

\section{F. Michael Christ}

University of California

Los Angeles, CA 90024-1555

christ@math.ucla.edu

Herbert Clemens

University of Utah

Salt Lake City, UT 84112

clemens@math.utah.edu
THOMAS ENRIGHT

University of California, San Diego

La Jolla, CA 92093

tenright@ucsd.edu

Nicholas ERCOLANI

University of Arizona

Tucson, AZ 85721

ercolani@math.arizona.edu

R. FINN

Stanford University

Stanford, CA 94305

finn@gauss.stanford.edu

VAUGHAN F. R. JONES

University of California

Berkeley, CA 94720

vfr@math.berkeley.edu
SteVen KerCKHofF

Stanford University

Stanford, CA 94305

spk@gauss.stanford.edu

MARTIN ScharLemanN

University of California

Santa Barbara, CA 93106

mgscharl@math.ucsb.edu

Harold STARK

University of California, San Diego

La Jolla, CA 92093

V. S. VARADARAJAN

University of California

Los Angeles, CA 90024-1555

vsv@math.ucla.edu

\section{SUPPORTING INSTITUTIONS}

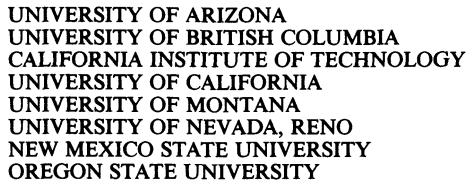

The Supporting Institutions listed above contribute to the cost of publication of this Journal, but they are not owners or publishers and have no responsibility for its content or policies.

Mathematical papers intended for publication in the Pacific Journal of Mathematics should be in typed form or offset-reproduced (not dittoed), double spaced with large margins. Please do not use built up fractions in the text of the manuscript. However, you may use them in the displayed equations. Underline Greek letters in red, German in green, and script in blue. The first paragraph must be capable of being used separately as a synopsis of the entire paper. In particular it should contain no bibliographic references. Please propose a heading for the odd numbered pages of less than 35 characters. Manuscripts, in triplicate, may be sent to any one of the editors. Please classify according to the 1991 Mathematics Subject Classification scheme which can be found in the December index volumes of Mathematical Reviews. Supply name and address of author to whom proofs should be sent. All other communications should be addressed to the managing editor, or Julie Honig, University of California, Los Angeles, California 90024-1555.

There are page-charges associated with articles appearing in the Pacific Journal of Mathematics. These charges are expected to be paid by the author's University, Government Agency or Company. If the author or authors do not have access to such Institutional support these charges are waived. Single authors will receive 75 free reprints; joint authors will receive a total of 100 free reprints. Additional copies may be obtained at cost in multiples of 50 .

The Pacific Journal of Mathematics (ISSN 0030-8730) is published monthly except for July and August. Regular subscription rate: $\$ 215.00$ a year (10 issues). Special rate: $\$ 107.50$ a year to individual members of supporting institutions.

Subscriptions, orders for numbers issued in the last three calendar years, and changes of address should be sent to Pacific Journal of Mathematics, P.O. Box 4163, Berkeley, CA 94704-0163, U.S.A. Old back numbers obtainable from Kraus Periodicals Co., Route 100, Millwood, NY 10546.

The Pacific Journal of Mathematics at University of California, c/o Department of Mathematics, 981 Evans Hall, Berkeley, CA 94720 (ISSN 0030-8730) is published monthly except for July and August. Second-class postage paid at Berkeley, CA 94704, and additional mailing offices. POSTMASTER: send address changes to Pacific Journal of Mathematics, P.O. Box 4163, Berkeley, CA 94704-0163.

PUBLISHED BY PACIFIC JOURNAL OF MATHEMATICS at University of California, Berkeley, CA 94720, A NON-PROFIT CORPORATION

This publication was typeset using $\mathcal{A} \mathcal{M} S-\mathrm{T}_{\mathrm{E}} \mathrm{X}$, the American Mathematical Society's $\mathrm{T}_{\mathrm{E}} \mathrm{X}$ macro system. Copyright (c) 1994 by Pacific Journal of Mathematics 


\section{PACIFIC JOURNAL OF MATHEMATICS}

Volume $162 \quad$ No. $2 \quad$ February 1994

On the existence of convex classical solutions to multilayer fluid

201 problems in arbitrary space dimensions

ANDREW FRENCH ACKER

Extremal functions and the Chang-Marshall inequality

VALENTIN V. ANDREEV and ALEC LANE MATHESON

Productive polynomials

RICHARD ARENS

On factor representations of discrete rational nilpotent groups and the 261 Plancherel formula

LAWRENCE JAY CORWIN and CAROLYN PFEFFER JOHNSTON

Commutants of Toeplitz operators on the Bergman space

ZELJKO CUCKOVIC

When $L^{1}$ of a vector measure is an AL-space

GUILlERMO P. CURBERA

A convexity theorem for semisimple symmetric spaces

305

KARL-HERMANN NEEB

Ideals of finite codimension in free algebras and the fc-localization

AMNON RosenmanN and ShMUEL Rosset

Dec groups for arbitrarily high exponents

BHARATH Al SETHURAMAN

Errata to: "The set of primes dividing the Lucas numbers has density $2 / 3$ "

JEFFREY C. LAGARIAS 